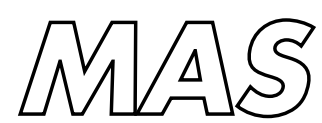

Modelling, Analysis and Simulation

Modelling, Analysis and Simulation

Algebraically decaying pulses in a Ginzburg-Landau system with a neutrally stable mode

A. Doelman, G. Hek, N. Valkhoff

RePort MAS-E0619 August 2006 
Centrum voor Wiskunde en Informatica (CWI) is the national research institute for Mathematics and Computer Science. It is sponsored by the Netherlands Organisation for Scientific Research (NWO).

CWI is a founding member of ERCIM, the European Research Consortium for Informatics and Mathematics.

CWI's research has a theme-oriented structure and is grouped into four clusters. Listed below are the names of the clusters and in parentheses their acronyms.

Probability, Networks and Algorithms (PNA)

Software Engineering (SEN)

\section{Modelling, Analysis and Simulation (MAS)}

Information Systems (INS)

Copyright (C) 2006, Stichting Centrum voor Wiskunde en Informatica

P.O. Box 94079, 1090 GB Amsterdam (NL)

Kruislaan 413, 1098 SJ Amsterdam (NL)

Telephone +31205929333

Telefax +31205924199

ISSN 1386-3703 


\title{
Algebraically decaying pulses in a Ginzburg-Landau system with a neutrally stable mode
}

\author{
ABSTRACT \\ In this paper, we study the existence and stability of pulse solutions in a system with interacting \\ instability mechanisms, that is described by a Ginzburg-Landau equation for an $A$-mode, \\ coupled to a diffusion equation for a $B$-mode. Our main question is whether this coupling may \\ stabilize solutions of the Ginzburg-Landau equation that are unstable when the interactions with \\ the neutrally stable $B$-mode are not included in the model. The spatially homogeneous $B$-mode \\ is supposed to be neutrally stable. This implies that the pulse solutions cannot decay \\ exponentially, but must decay with an algebraic rate as $x \rightarrow \infty$. As a consequence, the methods \\ that exist in the literature by which the stability of pulses in singularly perturbed reaction- \\ diffusion systems can be studied, need to be extended. This results in an 'algebraic NLEP \\ approach', that is expected to be relevant beyond the setting of this paper. As in the case of a \\ (weakly) stable $B$-mode [7], we establish by the application of this approach, that the $B$-mode \\ indeed introduces a mechanism that may stabilize pulses that are unstable when the \\ interactions with the $B$-mode are not taken into account.
}

2000 Mathematics Subject Classification: 35B35, 37L15, 35B32, 35K57,76E30

Keywords and Phrases: Ginzburg-Landau, stability of pulses, Evans function 



\title{
Algebraically decaying pulses in a Ginzburg-Landau system with a neutrally stable mode
}

\author{
Arjen Doelman*†, Geertje Hek $^{\dagger *}$, Nienke Valkhoff ${ }^{\dagger}$ \\ ${ }^{*}$ Centrum voor Wiskunde en Informatica \\ P.O. Box 94079, 1090 GB Amsterdam \\ the Netherlands \\ ${ }^{\dagger}$ Korteweg-de Vries Instituut, Universiteit van Amsterdam \\ Plantage Muidergracht 24, 1018 TV Amsterdam \\ the Netherlands
}

18th August 2006

\begin{abstract}
In this paper, we study the existence and stability of pulse solutions in a system with interacting instability mechanisms, that is described by a Ginzburg-Landau equation for an $A$-mode, coupled to a diffusion equation for a $B$-mode. Our main question is whether this coupling may stabilize solutions of the Ginzburg-Landau equation that are unstable when the interactions with the neutrally stable $B$-mode are not included in the model. The spatially homogeneous $B$-mode is supposed to be neutrally stable. This implies that the pulse solutions cannot decay exponentially, but must decay with an algebraic rate as $x \rightarrow \infty$. As a consequence, the methods that exist in the literature by which the stability of pulses in singularly perturbed reaction-diffusion systems can be studied, need to be extended. This results in an 'algebraic NLEP approach', that is expected to be relevant beyond the setting of this paper. As in the case of a (weakly) stable $B$-mode [7], we establish by the application of this approach, that the $B$-mode indeed introduces a mechanism that may stabilize pulses that are unstable when the interactions with the $B$-mode are not taken into account.
\end{abstract}

Mathematics Subject Classification: 35B35, 37L15, 35B32, 35K57,76E30.

\section{Introduction}

The leading order behavior of small perturbations in 'marginally unstable' systems of nonlinear partial differential equations defined on unbounded domains is generically determined by the complex Ginzburg-Landau equation (see [16] and the references therein). If the destabilization is caused by two competing instability mechanisms, the evolution of small solutions is determined by a system of coupled equations. Such a situation is a priori of co-dimension 2, but occurs very naturally in systems that have a spatially homogeneous neutrally stable mode. This mode appears in the linear stability analysis on which the derivation of the Ginzburg-Landau equations is based as a neither growing nor decaying structure that has $k_{c}=0$ as critical wave number. 
The homogeneous mode is diffusive, i.e., its evolution is governed by a real diffusion equation. In general, the coupled system that describes the interacting of a Ginzburg-Landau instability with a diffusive mode is given by

$$
\left\{\begin{aligned}
A_{t} & =\alpha_{1} A_{x x}+\alpha_{2} A+\alpha_{3}|A|^{2} A+\mu A B \\
\beta_{0} B_{t} & =\beta_{1} B_{x x}+G\left(B, B_{x},|A|^{2},|A|_{x}^{2},|A|_{x x}^{2}\right),
\end{aligned}\right.
$$

with $\alpha_{i} \in \mathbb{C}, i=1,2,3, A(x, t): \mathbb{R} \times \mathbb{R}^{+} \rightarrow \mathbb{C}, \mu, \beta_{0}, \beta_{1} \in \mathbb{R}, B(x, t): \mathbb{R} \times \mathbb{R}^{+} \rightarrow \mathbb{R}$, and $G$ a (real) function of $B, B_{x},|A|^{2},|A|_{x}^{2},|A|_{x x}^{2}$ that is in general nonlinear. Coupled equations of the type (1.1) appear in binary fluid convection [18, 19], biological/chemical systems [4], nematic liquid crystals [9], granular media [27], geophysical morphodynamics [14], systems with symmetries or conserved quantities $[3,15,17,28]$, etc. We refer to [20] for a survey of the appearance and relevance of systems of the form (1.1), and to [7] for more background on derivation of (1.1).

The precise structure of the function $G$ varies from case to case. In this paper, we focus on systems that are reversible in $x$, which implies that we assume that $G$ does not depend on $B_{x}$ or $|A|_{x}^{2}$. Moreover, we also assume that $G$ does not depend on $|A|_{x x}^{2}$ (these terms appear at leading order in systems with a conserved quantity $[15,28]$ ), and that it has the simple form

$$
G\left(B,|A|^{2}\right)=B g_{1}(B)+|A|^{2} g_{2}(B),
$$

with $g_{1}(0)=\alpha$ and $g_{2}(0)=\nu$ (see Remark 1.2). Note that $G$ 's of this form appear frequently in the literature. The stability of the diffusive, neutrally stable mode, sometimes called the Goldstone mode, is determined by $\alpha$. If $\alpha=0$, the $B$-mode is truly neutral, and it does not grow or decay in the linear analysis. In [7], we have considered the situation in which $\alpha$ is small and negative, but nonzero, so that the $B$-mode is 'weakly stable' and not 'fully neutral'. This enabled us to study the existence and stability of localized solutions to (a simplified, real version of) (1.1) by applying methods that have been developed in the context of reaction-diffusion equations. Note that also systems of the form (1.1) with $\alpha \neq 0$ appear quite naturally in the literature. In this paper, we consider the case of a completely neutral mode, i.e., we assume that $g_{1}(0)=0$ in (1.2). This means for the Taylor expansion of $g_{1}(B)$ that its first term is linear in $B$. This is also completely natural from the point of view of the derivation of (1.1) [7]. As a consequence, $G\left(B,|A|^{2}\right)$ varies quadratically in $B$ for small $B$ and $|A|^{2}=0$. This quadratic structure generates the algebraic, i.e. non-exponential, decay in the pulse solutions of (1.1).

The forthcoming analysis has two main themes.

The first theme concerns the issue of the impact of the neutral $B$-mode on the dynamics of the complex Ginzburg-Landau equation (i.e., the first equation in (1.1) with $B \equiv b_{0}$, a constant). This is especially relevant by the observations in $[18,19]$ and the references therein that indicate that unstable solutions of the Ginzburg-Landau equation can be stabilized by the interaction with a diffusive $B$-mode. It has been shown in [7] that the coupling of a 'weakly stable, almost neutral' $B$-mode to the Ginzburg-Landau equation for $A$ indeed may stabilize localized solutions of the Ginzburg-Landau equation that are unstable without this coupling.

In this paper we study this stabilization mechanism, now in the context of a Ginzburg-Landau equation coupled to a neutrally stable diffusive mode. As in [7], we consider a simplified version of (1.1),

$$
\left\{\begin{aligned}
A_{t} & =A_{x x}-A+A^{3}+\mu A B, \\
\varepsilon^{2} \tau B_{t} & =\varepsilon^{-2} B_{x x}-\varepsilon^{2} \eta B^{2}+\nu A^{2}+\beta A^{2} B .
\end{aligned}\right.
$$

Thus, we have assumed that the coefficients $\alpha_{i}, i=1,2,3$ are real and reduced the threecomponent model (1.1) - recall that $A(x, t) \in \mathbb{C}$ - to a two-component model by restricting to the case $A(x, t) \in \mathbb{R}$ (see Remark 1.3). Furthermore, we follow $[18,19]$ and focus on the situation in which the Ginzburg-Landau equation describes a subcritical bifurcation. We also 
have introduced the 'artificial' small parameter $0<\varepsilon \ll 1$ in the $B$-equation. As a consequence, $B_{x x}=\mathcal{O}\left(\varepsilon^{2}\right)$, and thus small. Since it is natural to assume that $B$ must be bounded, it (roughly) follows that $B=b_{0}$, a constant, at leading order in $\varepsilon$ (on long, but bounded spatial intervals). Thus, by introducing $\varepsilon$, we may indeed interpret (1.3) as a 'small perturbation' of its limit problem

$$
A_{t}=A_{x x}-\left(1-\mu b_{0}\right) A+A^{3},
$$

a subcritical scalar Ginzburg-Landau equation. Note that this equation has an unstable standing pulse solution (if $\mu b_{0}<1$, sections 2 and 3). Furthermore, we have set $g_{1}(B)=-\varepsilon^{2} \eta B$ and $g_{2}(B)=\nu+\beta B(1.2)$. We refer to Remark 1.1 for some further background on the model and its parameters. Notice that there are two differences between the central equation studied in [7] and (1.3). The main difference is the fact that the 'stable' linear term ' $-\varepsilon^{2} \alpha B$ ' of [7] is replaced by the 'neutral' quadratic term ' $-\varepsilon^{2} \eta B^{2}$ '. The second difference is that there is no ' $\sigma B_{x}$ ' term in (1.3) - see Remark 1.2.

As in [7], we will study the persistence and stability of the (unstable) homoclinic pulse solution of the (uncoupled, real) Ginzburg-Landau equation (1.4) as solution of the full system (1.3). The persistence problem corresponds to searching for standing waves in system (1.3), (Remark 1.2), i.e., to constructing heteroclinic or homoclinic orbits in the 4-dimensional singularly perturbed ODE,

$$
\left\{\begin{aligned}
a^{\prime} & =v, \\
v^{\prime} & =a-a^{3}-\mu a b, \\
b^{\prime} & =\varepsilon d, \\
d^{\prime} & =\varepsilon\left[\varepsilon^{2} \eta b^{2}-\nu a^{2}-\beta b a^{2}\right],
\end{aligned}\right.
$$

where the ' represents differentiation with respect to $x$. A physically relevant pulse solution satisfies $\lim _{x \rightarrow \pm \infty}|A(x, t)|=\lim _{x \rightarrow \pm \infty}|B(x, t)|=0$. Therefore, we look for homoclinic solutions $\gamma_{h}(x)$ to $(1.5)$, and corresponding pulse solutions $\left(A_{h}(x), B_{h}(x)\right)$, of (1.3), that satisfy $\lim _{x \rightarrow \pm \infty} \gamma_{h}(x)=(0,0,0,0)$. Due to the absence of the linear term in the $B$-equation, this critical point is non-hyperbolic/degenerate. Nevertheless, the methods of geometric singular perturbation theory can be applied, and the persistence issue can be settled in a relatively straightforward fashion (Theorem 2.1, Remark 1.3). However, the pulse $\left(A_{h}(x), B_{h}(x)\right)$ decays algebraically slow to $(0,0)$ as $|x| \rightarrow \infty$. This fact has a significant impact on the stability analysis, which brings us to the second main theme of this paper.

The linear stability analysis of localized solutions that do not decay exponentially fast to their 'background states' is the second main theme of this paper. This issue goes beyond the setting of the explicit model problems (1.1) and (1.3). Recently, there has been a growing interest in the stability analysis of localized solutions with algebraic decay. This issue appears naturally in various settings, such as the stability analysis of contact defects [23], absolute instabilities of pulses [24], and curvature effects on the spectra of spiral waves [25]. The present study can be seen as an extension of the general methods developed in $[5,6]$ to the case of pulses with algebraic decay. The approach developed here can be applied more general (singularly perturbed) systems.

In $[5,6]$, a method has been developed to decompose the Evans function $\mathcal{D}(\lambda)$ associated to the stability of $\left(A_{h}(x), B_{h}(x)\right)$ into a product of two transmission functions (in the context of singularly perturbed reaction-diffusion equations). The zeroes of $\mathcal{D}(\lambda)$, that correspond to the eigenvalues of the stability problem $[1,22]$, are shown to be determined by the zeroes of the second 'slow' transmission function $t_{2}(\lambda)$. Moreover, a method, called the NLEP method, is presented by which the $t_{2}(\lambda)$ can be computed explicitly. This (NLEP) approach has also been used in [7] to show that the 'weakly stable' $B$-mode can indeed stabilize unstable pulses in the GinzburgLandau equation. The fact that the pulses considered in $[5,6,7]$ decay exponentially is essential for the NLEP approach (both for the decomposition of $\mathcal{D}(\lambda)$ and for the computation of $t_{2}(\lambda)$ ). More specifically, in $[5,6,7]$, the $4 \times 4$ matrix $\mathcal{A}(x ; \lambda)$ that governs the stability of the pulse (see (3.3) and (3.4)) decays exponentially fast to a constant coefficient matrix $\mathcal{A}_{\infty}(\lambda)$. This matrix, 
the solutions to the associated constant coefficient problem, and the fact that $\left\|\mathcal{A}(x ; \lambda)-\mathcal{A}_{\infty}(\lambda)\right\|$ decays exponentially fast as $|x| \rightarrow \infty$, are fundamental ingredients of the NLEP approach. In this paper we extend the NLEP approach by introducing an 'intermediate' matrix $\mathcal{A}_{\text {alg }}(x ; \lambda)$ in such a way that $\left\|\mathcal{A}(x ; \lambda)-\mathcal{A}_{\text {alg }}(x ; \lambda)\right\|$ decays exponentially fast, while $\mathcal{A}_{\text {alg }}(x ; \lambda)$ only decays algebraically slow to $\mathcal{A}_{\infty}(\lambda)$. The role of the fundamental solutions of the constant coefficient problem associated to $\mathcal{A}_{\infty}(\lambda)$ is now played by the (four, independent) solutions of the linear system associated to $\mathcal{A}_{\text {alg }}(x ; \lambda)$. These solutions can be determined/approximated explicitly by applying the WKBJ method and solving a Whittaker equation (see also [25]).

These solutions also form the backbone of the extension of the NLEP approach to pulses that decay algebraically. The Evans function can again be shown to be decomposable, and its zeroes are all determined by the zeroes of an explicitly computable 'slow' transmission function $t_{b}(\lambda)$. However, both the analysis and its outcome are more involved than in the standard, exponential case. Moreover, it is found that the Evans function has a pole of order 2 at $\lambda=0$, the tip of the essential spectrum.

It should be noted that the extension of the NLEP approach developed here can also be expected to be relevant beyond the setting of pulses that decay algebraically. The methods developed in $[5,6]$ are based on the decomposition of the stability problem into a fast system and a 'trivial' slow system (of constant coefficient type, associated to $\mathcal{A}_{\infty}(\lambda)$ ). In the class of systems considered in [5, 6] (and in [7]) this slow system is indeed 'trivial', but it isn't in the context of algebraically decaying pulses, and, the slow limit system also is not trivial in the stability analysis of fronts (with exponential decay!) in the class of systems considered in [8]. The analysis of [8] implies that the slow limit system associated to the stability problem of a pulse is in general not trivial, i.e., not of constant coefficients type. This feature appears to be generic within a large class of reaction-diffusion problems. The methods developed here are expected to be applicable too in this context.

In the final section of this paper, we return to its first main theme and apply the 'algebraic NLEP approach' to the stability problem of the pulse $\left(A_{h}(x), B_{h}(x)\right)$. As in [7], we first consider a linear $G$, i.e., $g_{2}(B)=\nu$ in (1.2), or $\beta=0$ in (1.3). It is shown that the neutral mode does have an $\mathcal{O}(1)$ effect on the eigenvalues of the associated limit problem - the unstable pulse solution of (1.4). Nevertheless, the pulse $\left(A_{h}(x), B_{h}(x)\right)$ remains unstable (Theorem 4.3). When we introduce higher order terms in the $B$-equation, we need to distinguish between the 6 different cases that appear from the existence theory (Lemma 2.3). In the first 4 cases, $\left(A_{h}(x), B_{h}(x)\right)$ is unstable (Theorem 4.8), however, it is shown that the neutral $B$ mode can indeed stabilize an unstable pulse (by a Hopf bifurcation) and that $\left(A_{h}(x), B_{h}(x)\right)$ may become stable in the final two cases (Theorems 4.11 and 4.12).

Although the analysis of the transmission function $t_{b}(\lambda)$ is more involved, and in fact more complete, than that in [7], it is somewhat surprising to notice that, in the end, the stabilization mechanism in the case with a neutrally stable $B$-mode acts in a way that is remarkably similar to that of [7] in which the $B$-mode was weakly stable. This especially holds for the nature of the stabilization - a Hopf bifurcation - and the fact that $\left(A_{h}(x), B_{h}(x)\right)$ can only be stabilized in the presence of higher order nonlinear terms in $G\left(B,|A|^{2}\right)$.

Remark 1.1 We assume that $\tau>$ and that (1.3) is fully coupled, i.e., that $\mu \neq 0$. We also assume that $\eta \neq 0$, since the presence of the $B^{2}$-term in (1.3) is crucial to the analysis (although a similar analysis can be performed with a cubic, or higher order, nonlinearity). By the symmetry $\{(B, \mu, \eta, \nu) \rightarrow(-B,-\mu,-\eta,-\nu)\}$ of $(1.3)$, we can thus restrict our attention to the case $\eta>0$. The motivation to assume that both the $B_{t^{-}}$and the $B^{2}$-terms in $(1.3)$ are of $\mathcal{O}\left(\varepsilon^{2}\right)$ is mostly mathematical. For instance, the transitions from instability to stability occur for these scalings of $\beta_{0}(1.1)$ and $g_{1}(B)(1.2)$. 
Remark 1.2 As opposed to [7], where a symmetry-breaking term $\sigma B_{x}$ was introduced in the $B$-equation, system (1.3) is symmetric under $\{x \rightarrow-x\}$. This symmetry is inherited by (1.5),

$$
\{(x, v, d) \rightarrow(-x,-v,-d)\}
$$

Due to the reversibility symmetry, one typically expects to find standing wave solutions in (1.3). Thus, we focus on the most simple persistence/existence problem and reduce (1.3) to (1.5) in section 2. As in [7], our methods can also be applied to the setting of (slowly) travelling waves. In fact, our methods can be applied to systems (1.1) in which the function $G$ is less simple than in (1.2) or (1.3). Finally, we note that (1.3) is also symmetric under $\{A \rightarrow-A\}$. The pulse solutions $\left(A_{h}(x), B_{h}(x)\right)$ studied in this paper have $A_{h}(x)>0$ for all $x \in \mathbb{R}$. By the symmetry, our results also hold for the negative pulses $\left(-A_{h}(x), B_{h}(x)\right)$.

Remark 1.3 The most restrictive simplification in the reduction of (1.1) to (1.3) is the assumption that $A(x, t) \in \mathbb{R}$, instead of $A(x, t) \in \mathbb{C}$. In [11], the persistence issue is considered for the complex case. Unlike in the reduction to $A(x, t) \in \mathbb{R}([7]$, section 2$)$, the existence/persistence issue becomes a challenging problem. Nevertheless, the persistence of pulse solutions to the complex Ginzburg-Landau equation under coupling with slow diffusion can be established. Whether these pulses can be stable, is the subject of further analysis.

\section{Persistence}

In this section, we study the existence of stationary pulse solutions to (1.3). The four dimensional 'fast' system (1.5) can alternatively be written as the slow system

$$
\left\{\begin{aligned}
\varepsilon \dot{a} & =v \\
\varepsilon \dot{v} & =a-a^{3}-\mu a b \\
\dot{b} & =d, \\
\dot{d} & =\varepsilon^{2} \eta b^{2}-\nu a^{2}-\beta b a^{2}
\end{aligned}\right.
$$

Here the dot denotes the derivative with respect to the slow variable $\xi=\varepsilon x$. In the forthcoming stability analysis it will be useful to explicitly separate the $x$-regions in which the (spatial) dynamics are slow or fast. Therefore, we introduce the fast spatial region

$$
I_{f}=\left[-\frac{1}{\sqrt{\varepsilon}}, \frac{1}{\sqrt{\varepsilon}}\right] .
$$

The boundary of $I_{f}$ is clearly in the transition zone between the slow $\xi$ - and the fast $x$-scale, i.e., $|\xi| \ll 1$ and $|x| \gg 1$ at $\partial I_{f}$. This condition does not define $I_{f}$ uniquely, nevertheless, (2.2) is also used as the standard choice for $I_{f}$ in $[5,8,7]$.

\subsection{The reduced fast and slow systems}

Putting $\varepsilon=0$ in (1.5), we obtain the associated fast reduced system,

$$
\left\{\begin{array}{l}
a^{\prime}=v \\
v^{\prime}=\left(1-\mu b_{0}\right) a-a^{3} \\
b^{\prime}=0 \\
d^{\prime}=0
\end{array}\right.
$$


Hence, solutions of (2.3) satisfy $b \equiv b_{0}, d \equiv d_{0} ;(2.3)$ is a two-parameter family of planar, integrable systems (Figure 1). We assume throughout this paper that

$$
1-\mu b_{0}>0
$$

so that (2.3) has a family of (positive) homoclinic solutions

$$
\begin{aligned}
& a_{0}\left(x ; b_{0}\right)=\sqrt{2\left(1-\mu b_{0}\right)} \operatorname{sech}\left(\sqrt{1-\mu b_{0}} x\right) \\
& v_{0}\left(x ; b_{0}\right)=a_{0}^{\prime}\left(x ; b_{0}\right)
\end{aligned}
$$

that satisfy $\lim _{x \rightarrow \pm \infty}\left(a_{0}\left(x ; b_{0}\right), v_{0}\left(x ; b_{0}\right)\right)=(0,0)$ (Remark 1.2). These orbits correspond to pulse solutions $A(x, t)=a_{0}\left(x ; b_{0}\right)$ in the uncoupled Ginzburg-Landau equation (1.4). These pulses may persist in various forms as solution to the full equation, such as wave trains of multi-pulse patterns ([5] and the references therein). Here, as in [7], we restrict our attention to the most simple setting and only consider solutions of (1.3) that satisfy $\lim _{x \rightarrow \pm \infty}|A(x, t)|=\lim _{x \rightarrow \pm \infty}|B(x, t)|=0$. In other words, we look for homoclinic solutions $\gamma_{h}(x)$ to (1.5) that satisfy $\lim _{x \rightarrow \pm \infty} \gamma_{h}(x)=S$, where we define $S$ as the degenerate fixed point $(0,0,0,0)$ of $(1.5)$.

System (2.3) possesses three 2-dimensional invariant manifolds $\left\{a_{0}=0, v_{0}=0\right\}$ and $\left\{a_{0}=\right.$ $\left.\pm \sqrt{1-\mu b_{0}}, v_{0}=0\right\}$. Only the first one, $\mathcal{M}_{0}:=\left\{\left(a_{0}, v_{0}, b_{0}, d_{0}\right) \mid a_{0}=v_{0}=0\right\}$, is filled with hyperbolic equilibria, and is thus a normally hyperbolic invariant manifold $[10,13] ; \mathcal{M}_{0}$ has 3 -dimensional stable and unstable manifolds $W^{s}\left(\mathcal{M}_{0}\right)$ and $W^{u}\left(\mathcal{M}_{0}\right)$, which are the unions of the 2-parameter families of one-dimensional stable and unstable manifolds of the saddle points $\left(0,0, b_{0}, d_{0}\right) \in \mathcal{M}_{0}$ of $(2.3)$. For each $\left(b_{0}, d_{0}\right)$ satisfying $(2.4), \mathcal{M}_{0}$ is connected to itself by the homoclinic orbit given by (2.5). The family of homoclinic orbits forms a 3 -dimensional homoclinic manifold $\mathcal{H}=W^{s}\left(\mathcal{M}_{0}\right) \cap W^{u}\left(\mathcal{M}_{0}\right)$.

If $\varepsilon$ is sufficiently small, geometric singular perturbation theory $[10,13]$ guarantees that there exists a locally invariant manifold $\mathcal{M}_{\varepsilon}$ for the perturbed system (1.5) which is diffeomorphic to and $\mathcal{O}(\varepsilon) C^{1}$-close to $\mathcal{M}_{0}$. Clearly, $\mathcal{M}_{\varepsilon}=\mathcal{M}_{0}$ in the current setting. In addition, the theory states that for $0<\varepsilon \ll 1$ the manifold $\mathcal{M}_{\varepsilon}$ has three-dimensional stable and unstable manifolds $W^{s}\left(\mathcal{M}_{\varepsilon}\right)$ and $W^{u}\left(\mathcal{M}_{\varepsilon}\right)$ that are again $\mathcal{O}(\varepsilon) C^{1}$-close and diffeomorphic to their counterparts $W^{s}\left(\mathcal{M}_{0}\right)$ and $W^{u}\left(\mathcal{M}_{0}\right)$.

The flow on $\mathcal{M}_{\varepsilon}$ for the full system (1.5) can be determined by substituting $(a, v)=(0,0)$. The resulting 2-dimensional system,

$$
\left\{\begin{aligned}
b^{\prime} & =\varepsilon d, \\
d^{\prime} & =\varepsilon^{3} \eta b^{2},
\end{aligned}\right.
$$

has one critical point, $(b, d)=(0,0)$, that corresponds to $S$ in the 4-dimensional system (1.5). Due to the fact that there is no linear $B$ term in $(1.3),(0,0)$ is degenerate, the flow generated by (2.6) on $\mathcal{M}_{\varepsilon}$ has a cusp at $(0,0)$. Nevertheless, $S$ has stable and unstable manifolds, and their restrictions to $\mathcal{M}_{\varepsilon}$ together form the cusp (see also Figure 2). However, due to this degeneracy, the solutions on $W^{s}(S)$, respectively $W^{u}(S)$, do not converge exponentially to $S$ as $x \rightarrow \infty$, resp. $x \rightarrow-\infty$. The slow flow (2.6) can be integrated to obtain formulas for the corresponding orbits in $\mathcal{M}_{\varepsilon}$, the integral being

$$
K=d^{2}-\frac{2 \varepsilon^{2} \eta}{3} b^{3} .
$$

The stable and unstable manifolds of $S$ (restricted to $\mathcal{M}_{\varepsilon}$ ) correspond to $K=0$, and are given by

$$
w^{u, s}=\left.W^{u, s}(0,0)\right|_{\mathcal{M}_{\varepsilon}}=\left\{(b, d) \mid d= \pm \frac{\varepsilon}{3} \sqrt{6\left|\eta b^{3}\right|}\right\} .
$$

Note that the curves $w^{u}$ and $w^{s}$ are $\mathcal{O}(\varepsilon)$ apart by the scaling of the $B^{2}$-term in (1.3). As in [7], this is necessary for establishing the existence of the pulse solution of our interest. The curves 


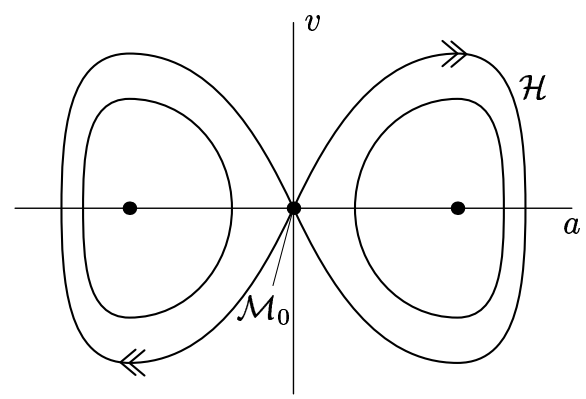

Figure 1: The $\left(a_{0}, v_{0}\right)$ phase space of the fast subsystem.

$w^{u, s}$ can be parameterized by the slowly varying algebraically decaying exact solutions

$$
b_{\text {alg }}\left(x ; X^{*}\right)=b_{\text {alg }}(x ; b(0))=\frac{6}{\eta\left(X^{*}+\varepsilon^{2} x\right)^{2}}=\frac{b(0)}{\left(1+\varepsilon^{2} x \sqrt{\eta b(0) / 6}\right)^{2}}
$$

of $(2.6)$, where $b_{\text {alg }}(x)$ is parameterized either by its value $b(0)$ at $x=0$, or by its blow-up point $x=-X^{*} / \varepsilon^{2}$, that are related by

$$
X^{*}=\sqrt{\frac{6}{\eta b(0)}}>0 .
$$

Note that $b_{\text {alg }}(x)$ varies as a function of the super-slow spatial variable $X=\varepsilon^{2} x$ and thus does not change (at leading order) on spatial intervals of length $\mathcal{O}(1 / \sqrt{\varepsilon})$, i.e., $b_{\text {alg }}(x)=b(0)$ (at leading order) for $x \in I_{f}$ (2.2). Note also that our choice $\eta>0$ forces $b(0)>0$ (Remark 1.1) and that $w^{s}$, respectively $w^{u}$, is described by $b_{\text {alg }}(x)$ for $x>-X^{*} / \varepsilon^{2}$, resp. by $b_{\text {alg }}(-x)$ for $x<X^{*} / \varepsilon^{2}$ (1.6).

We can now formulate our main existence (or persistence) result.

Theorem 2.1 Let $\eta>0, \mu \neq 0$ and $\varepsilon>0$ be sufficiently small.

(i) If $\beta=0$ and $\nu>0$, then (1.5) has a uniquely determined homoclinic solution $\gamma_{h}(x)=$ $\left(a_{h}(x), v_{h}(x), b_{h}(x), d_{h}(x)\right)$ to $S=(0,0,0,0)$.

(ii) If $\beta \neq 0$, then there are open regions in $(\mu, \nu, \eta, \beta)$-space in which system (1.5) possesses 0 , 1 , or 2 (different) homoclinic orbits $\gamma_{h}(x)$ to $S=(0,0,0,0)$. The boundary between the regions with 0 or 2 homoclinic orbits is formed by a co-dimension 1 manifold of homoclinic saddle-node bifurcations.

In all cases,

$$
\left|a_{h}(x)-a_{0}\left(x ; b_{0}\right)\right|,\left|v_{h}(x)-v_{0}\left(x ; b_{0}\right)\right|,\left|b_{h}(x)-b_{0}\right|,\left|d_{h}(x)\right|=\mathcal{O}(\varepsilon)
$$

uniformly for $x \in I_{f}$, with $a_{0}\left(x ; b_{0}\right), v_{0}\left(x ; b_{0}\right)$ as in (2.5), and $b_{0}$ a solution of

$$
\frac{1}{3} \sqrt{6 \eta b_{0}^{3}}=2\left(\nu+\beta b_{0}\right) \sqrt{1-\mu b_{0}} .
$$

that satisfies (2.4). Moreover, there are $\mathcal{O}(1)$ constants $C, K>0$ such that for $x \in \mathbb{R} \backslash I_{f}$,

$$
\left|a_{h}(x)\right|,\left|v_{h}(x)\right|,\left|b_{h}(x)-b_{\mathrm{alg}}(|x| ; b(0))\right|,\left|\varepsilon d_{h}(x)-\frac{d}{d x} b_{\mathrm{alg}}(|x| ; b(0))\right|<C e^{-K|x|},
$$

with $b(0)=b_{0}+\mathcal{O}(\varepsilon)$ and $b_{\mathrm{alg}}(x ; b(0))$ as in (2.9). The orbits $\gamma_{h}(x)$ correspond to standing pulse solutions $\left(A_{h}(x), B_{h}(x)\right)=\left(a_{h}(x), b_{h}(x)\right)$ of (1.3) that decay algebraically to $(0,0)$ as $|x| \rightarrow \infty$. 
In [7], similar results have been proved for a coupled Ginzburg-Landau-diffusion equation with linear decay in the $B$-equation. The situation there is standard, since the flow on $\mathcal{M}_{\varepsilon}$ is nondegenerate and linear. Nevertheless, apart from the algebraic decay of the $B$-component, the persistence results for (1.3) are qualitatively remarkably similar to those in [7]: if $\beta=0$, i.e., if there are no higher order terms in the $B$-equation, then there is a uniquely determined homoclinic solution $\gamma_{h}(x)$ to $(0,0,0,0)$ in $(1.5)$. This uniqueness is lost, and homoclinic saddle-node bifurcation occur if $\beta \neq 0$. Quantitatively, there are of course differences. We refer to Lemma 2.3 for a more detailed description of the open regions in $(\mu, \nu, \eta, \beta)$-space mentioned in Theorem 2.1 (ii). We will consider each of the 6 cases discussed in this lemma in detail in the stability analysis (section 4.2 ).

\subsection{The proof of Theorem 2.1}

Although the critical point $S \subset \mathcal{M}_{\varepsilon}$ is degenerate, the slow manifold $\mathcal{M}_{\varepsilon}$ itself is normally hyperbolic. This fact is crucial in the proof of Theorem 2.1, since it allows us to apply the standard machinery of geometric singular perturbation theory.

The stable and unstable manifolds $W^{s}\left(\mathcal{M}_{\varepsilon}\right)$ and $W^{u}\left(\mathcal{M}_{\varepsilon}\right)$ no longer merge in a homoclinic manifold $\mathcal{H}$ if $\varepsilon \neq 0$, but may intersect in one or more 2-dimensional surfaces. If homoclinic orbits to the point $S$ exist, they must lie in one of these intersections. Since the system (1.5) is an $\mathcal{O}(\varepsilon)$ perturbation of an integrable system with periodic orbits inside $\mathcal{H}$, components of $W^{u}\left(\mathcal{M}_{\varepsilon}\right)$ and $W^{s}\left(\mathcal{M}_{\varepsilon}\right)$ inside $\mathcal{H}$ wind around and intersect the hyperplane $\{v=0\}$ several times. Adiabatic Melnikov theory [21] provides a measure $\Delta\left(b_{0}, d_{0}\right)$ that determines the leading order distance (divided by $\varepsilon$ ) between the (first) intersections of $W^{u}\left(\mathcal{M}_{\varepsilon}\right)$ and $W^{s}\left(\mathcal{M}_{\varepsilon}\right)$ with $\{v=0\}$,

$$
\Delta\left(b_{0}, d_{0}\right)=\int_{-\infty}^{\infty} \mu d_{0} a_{0}\left(x ; b_{0}\right) v_{0}\left(x ; b_{0}\right) x d x
$$

with $a_{0}\left(x ; b_{0}\right), v_{0}\left(x ; b_{0}\right)$ as in $(2.5)$. The zeroes of $\Delta\left(b_{0}, d_{0}\right)$ give the intersections of $W^{u}\left(\mathcal{M}_{\varepsilon}\right)$ and $W^{s}\left(\mathcal{M}_{\varepsilon}\right)$. The integrand in (2.14) is an even function of $x$, thus, since $\mu \neq 0$, these intersections must have $d_{0}=0$ (the integral can also be explicitly computed [7]). A priori this is only a leading order result, however it follows by the reversibility symmetry (1.6) and the fact that $W^{u, s}\left(\mathcal{M}_{\varepsilon}\right)$ are $\mathcal{O}(\varepsilon) C^{1}$-close to $W^{u, s}\left(\mathcal{M}_{0}\right)$ that

$$
W^{u}\left(\mathcal{M}_{\varepsilon}\right) \cap W^{s}\left(\mathcal{M}_{\varepsilon}\right) \cap\{v=0\} \subset\{d=0\} .
$$

This determines a one-parameter family of orbits biasymptotic to $\mathcal{M}_{\varepsilon}$. For any such orbit $\gamma\left(x ; x_{0}\right)$, i.e., with initial condition $x_{0}=\gamma\left(0 ; x_{0}\right) \in W^{u}\left(\mathcal{M}_{\varepsilon}\right) \cap W^{s}\left(\mathcal{M}_{\varepsilon}\right) \cap\{v=0\}$ there exist two orbits $\gamma^{ \pm}\left(x ; x_{0}^{ \pm}\right) \subset \mathcal{M}_{\varepsilon}$ such that $\left\|\gamma\left(x ; x_{0}\right)-\gamma^{+}\left(x ; x_{0}^{+}\right)\right\|$, respectively $\left\|\gamma\left(x ; x_{0}\right)-\gamma^{-}\left(x, x_{0}^{-}\right)\right\|$, is exponentially small in $\varepsilon$ and $x$ for $x \geq 1 / \sqrt{\varepsilon}$, resp. $x \leq-1 / \sqrt{\varepsilon}$. An orbit $\gamma_{h}(x)=\gamma\left(x ; x_{0}\right)$ is homoclinic to $S \in \mathcal{M}_{\varepsilon}$ if its base point $x_{0}^{+}$satisfies $x_{0}^{+} \subset w^{s}(2.8)$, with $w^{s}$ the stable manifold of $S$ on $\mathcal{M}_{\varepsilon}$. This automatically implies by (1.6) that $x_{0}^{-} \subset w^{u}$. In Figure $2, \gamma_{h}(x)=\gamma\left(x ; x_{0}\right)$ is sketched, together with its initial condition $x_{0}=\gamma\left(0, x_{0}\right) \in W^{u}\left(\mathcal{M}_{\varepsilon}\right) \cap W^{s}\left(\mathcal{M}_{\varepsilon}\right) \cap\{v=0\}$ and its base points $x_{0}^{ \pm} \in \mathcal{M}_{\varepsilon}$.

The take off curve $T_{o} \subset \mathcal{M}_{\varepsilon}$, respectively touch down curve $T_{d} \subset \mathcal{M}_{\varepsilon}$, represents the collection of base points of the solutions in $W^{u}\left(\mathcal{M}_{\varepsilon}\right)$, resp. $W^{s}\left(\mathcal{M}_{\varepsilon}\right)$ that are asymptotic to $\mathcal{M}_{\varepsilon}$ as $x \rightarrow \infty$, resp. $x \rightarrow-\infty$. Hence,

$$
T_{o}:=\bigcup_{x_{0}}\left\{x_{0}^{-}=\gamma^{-}\left(0, x_{0}^{-}\right)\right\}, \quad T_{d}:=\bigcup_{x_{0}}\left\{x_{0}^{+}=\gamma^{+}\left(0, x_{0}^{+}\right)\right\},
$$

where the unions are over all $x_{0} \in W^{s}\left(\mathcal{M}_{\varepsilon}\right) \cap W^{u}\left(\mathcal{M}_{\varepsilon}\right) \cap\{v=0\}$. The sets $T_{o}$ and $T_{d}$ can be determined by computing the accumulated change in $b$ and $d$ of $\gamma\left(x ; x_{0}\right) \subset W^{u}\left(\mathcal{M}_{\varepsilon}\right) \cap W^{s}\left(\mathcal{M}_{\varepsilon}\right)$ 


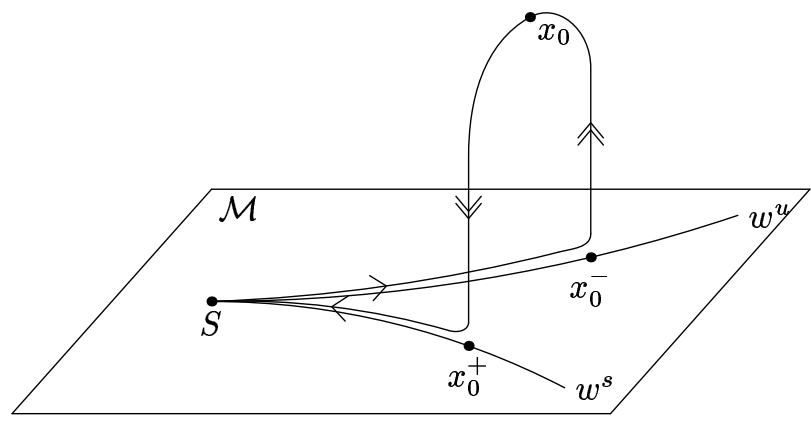

Figure 2: Sketch of a homoclinic solution $\gamma_{h}(x)$ as given by Theorem 2.1.

during half a circuit through the fast field (using (1.5), (2.3), (2.5) - see [5, 7]. Since the degeneracy of $S$ does not appear at leading order in the fast field, the calculations are identical to those in [7] and result in

$$
T_{o}=\left\{\left(b_{0}, d_{0}^{-}\right) \mid d_{0}^{-}=2 \varepsilon\left(\nu+\beta b_{0}\right) \sqrt{1-\mu b_{0}}\right\},
$$

up to corrections of $\mathcal{O}\left(\varepsilon^{2}|\log \varepsilon|\right) ; T_{d}=\left\{\left(b_{0}, d_{0}^{+}\right) \mid d_{0}^{+}=-d_{0}^{-}\right\}$, by the symmetry (1.6).

The orbit $\gamma\left(x ; x_{0}\right) \subset W^{u}\left(\mathcal{M}_{\varepsilon}\right) \cap W^{s}\left(\mathcal{M}_{\varepsilon}\right)$ is homoclinic to $S$ if its base point $x_{0}^{-}$, given by (2.16), is on $w^{u}$, i.e., if $T_{o}$ intersects $w^{u}$ and thus if (2.12) in Theorem 2.1 holds. By (1.6), this immediately implies that also $T_{d} \cap w^{s} \neq \emptyset$. The following two lemmas are obtained by a straightforward analysis of (2.12) and give more quantitative information on parts (i) and (ii) of Theorem 2.1.

Lemma 2.2 Let $\mu \neq 0, \nu>0, \eta>0, \beta=0$, and . $\varepsilon$ sufficiently small: (2.12) determines a unique value $b_{0}(\mu, \nu)$ at which $T_{o}$ intersects $w^{u}$ transversally.

Lemma 2.3 Let $\beta \neq 0, \eta>0$, and $\varepsilon>0$ be sufficiently small. The number of transversal intersections between $w^{u}$ and $T_{o}$ depends on the parameters $\mu, \nu, \beta$ and $\eta>0$ in the following way:

(i) If $\mu>0, \nu<0, \beta \leq-\mu \nu$ or $\mu<0, \nu<0, \beta<0$, then $T_{o} \cap w^{u}=\emptyset$ for all $\eta$.

(ii) If $\mu>0, \nu>0$ or $\mu<0, \nu>0, \beta<0$, then $T_{o} \cap w^{u}$ consists of one point for each $\eta$.

(iii) If $\mu>0, \nu<0, \beta>-\mu \nu$, then there exists an $\eta_{s n}>0$ such that $T_{o} \cap w^{u}$ contains two points for $\eta<\eta_{s n}, T_{o} \cap w^{u}=\emptyset$ for $\eta>\eta_{s n}$.

(iv) If $\mu<0, \nu>0, \beta>0$, then there exists an $\eta_{\ell}>0$, with $\eta_{\ell}=-6 \beta^{2} \mu+\mathcal{O}(\varepsilon)$, such that $T_{o} \cap w^{u}=\emptyset$ for $\eta<\eta_{\ell} ; T_{o} \cap w^{u}$ consists of one point for $\eta>\eta_{\ell}$.

(v) If $\mu<0, \nu<0, \beta>0$ and $\beta-2 \mu \nu>0$, then there exists an $\eta_{\ell}>0, \eta_{\ell}=-6 \beta^{2} \mu+\mathcal{O}(\varepsilon)$ and an $\eta_{s n}>\eta_{\ell}>0$, such that $T_{o} \cap w^{u}$ consists of one point for $\eta<\eta_{\ell}$, and of two points for $\eta_{\ell}<\eta<\eta_{s n} ; T_{o} \cap w^{u}=\emptyset$ for $\eta>\eta_{s n}$.

(vi) If $\mu<0, \nu<0, \beta>0$ and $\beta-2 \mu \nu<0$, then there exists an $\eta_{\ell}>0, \eta_{\ell}=-6 \beta^{2} \mu+\mathcal{O}(\varepsilon)$, such that $T_{o} \cap w^{u}$ consists of one point for $\eta<\eta_{\ell} ; T_{o} \cap w^{u}=\emptyset$ for $\eta>\eta_{\ell}$. 
The relative positions of $T_{o}$ and $w^{u}$ are sketched in Figures 3 for each of the 6 cases. Note that we have for simplicity decided to not consider the transitional cases such as $\nu=0, \beta-2 \mu \nu=0$, etc. in the statement of this lemma. The following lemma gives a more quantitative description of the non-transversal intersection that occurs at a saddle-node bifurcation (see also Section 3.5).

Lemma 2.4 The intersections $T_{o} \cap l^{u}$ and $T_{d} \cap l^{s}$ are non-transversal for parameter combinations at which both (2.12) and

$$
\frac{1}{2} \sqrt{6 \eta b_{0}}=-\frac{\mu\left(\nu+\beta b_{0}\right)}{\sqrt{1-\mu b_{0}}}+2 \beta \sqrt{1-\mu b_{0}}
$$

hold (at leading order). For these parameter combinations, a saddle-node bifurcation of homoclinic orbits takes place. Such a saddle-node bifurcation cannot occur if $\beta=0$.

The existence of an homoclinic orbit $\gamma_{h}(x) \subset W^{u}\left(\mathcal{M}_{\varepsilon}\right) \cap W^{s}\left(\mathcal{M}_{\varepsilon}\right)$ now follows from the above geometrical construction in combination with the Melnikov method and the quantitative results on the relative positions of $T_{o}$ and $w^{u}$ of Lemmas 2.2 and 2.3. Since the arguments are in essence identical to those in (for instance) $[5,7]$ we refrain from giving more details.

The approximations (2.11) and (2.13) of the components $\left(a_{h}(x), v_{h}(x), b_{h}(x), d_{h}(x)\right)$ in Theorem 2.1 both follow immediately from the asymptotic construction of $\gamma_{h}(x) ;(2.11)$ is based on the standard - see $[5,7]$ - approximations in the fast field $I_{f}$, i.e., the part of $\gamma_{h}(x)$ that is not exponentially close to the slow manifold $\mathcal{M}_{\varepsilon}$.

A direct application of Fenichel theory yields that all orbits in $W^{u}\left(\mathcal{M}_{\varepsilon}\right) \cap W^{s}\left(\mathcal{M}_{\varepsilon}\right)$ are exponentially close to $\mathcal{M}_{\varepsilon}$ outside $I_{f}$. This is quantitatively described by the estimates on $a_{h}(x)$ and $v_{h}(x)$ in (2.13). The take off point associated to $\gamma_{h}(x)$ is, by construction, on $w^{u}$ (and, by (1.6), its touch down point on $\left.w^{s}\right)$. Thus, for $x \in \mathbb{R} \backslash I_{f}, \gamma_{h}(x)$ must be exponentially close to either $w^{u} \subset \mathcal{M}_{\varepsilon}$ or $w^{s} \subset \mathcal{M}_{\varepsilon}$. Note that this observation is completely independent of the (singular) nature of the stable and unstable manifold $w^{s, u}$ of $S$. The slow manifold $\mathcal{M}_{\varepsilon}$ is exactly given by $\{a, v, b, d): a=v=0\}$, thus, the flow on $\mathcal{M}_{\varepsilon}$ is determined by $(2.6)$, without any higher order corrections in $\varepsilon$. Therefore, $w^{s, u}$ are exactly given by the explicit solution $b_{\mathrm{alg}}(x ; b(0))$ in which $b(0)$ is the only free parameter left. Hence, the estimates on $b_{h}(x)$ and $d_{h}(x)$ in $(2.13)$ follow. The construction of $\gamma_{h}(x)$ automatically yields the leading order approximation $b(0)=b_{0}$ (with $b_{0}$ determined by $(2.12)$ ).

\section{$3 \quad$ Linear stability analysis}

The linearized stability of the homoclinic pulse solutions $\left(A_{h}(x), B_{h}(x)\right)$ of $(1.3)$ can be determined by an Evans function method. However, since the pulse $\left(A_{h}(x), B_{h}(x)\right)$ does not converge exponentially to $(0,0)$ as $x \rightarrow \pm \infty$, we need to adapt the standard approach. Nevertheless, we can use the fact that the constructed single pulse solutions consist of a fast part, that decays on an exponential scale, and two slow parts close to the slow manifold for which the decay is only algebraic, to establish a decomposition of the Evans function $\mathcal{D}(\lambda)$ into a product of two explicitly computable transmission functions $t_{a}(\lambda)$ and $t_{b}(\lambda)$. The fact that $B_{h}(x)$ does not decay exponentially is especially encoded in $t_{b}(\lambda)$, that will have a much more complex structure than in the standard exponential case [5,7]. Moreover, we will find that $\mathcal{D}(\lambda)$ has a pole of order 2 at $\lambda=0$, while it is smooth (and non-zero) near $\lambda=0$ in the exponential case. 


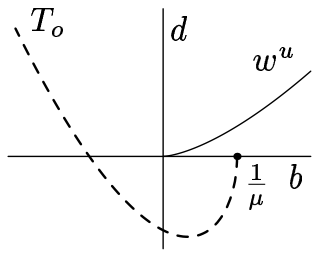

(i)

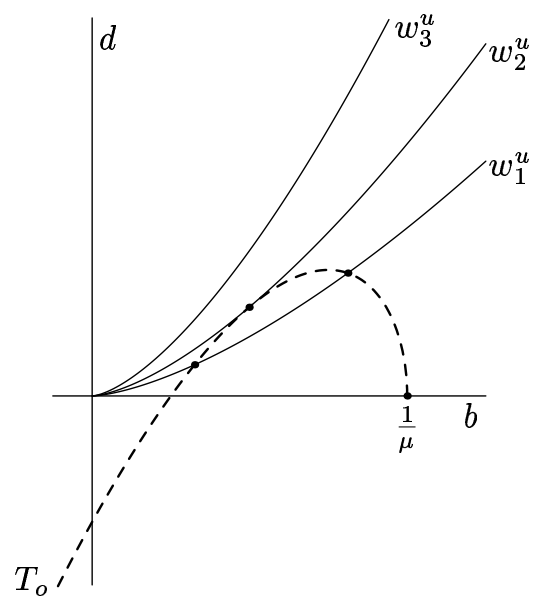

(iii)

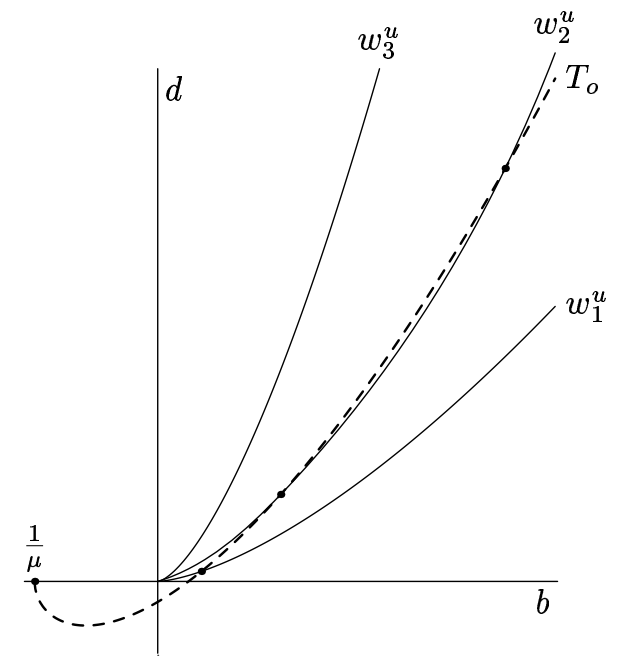

(v)

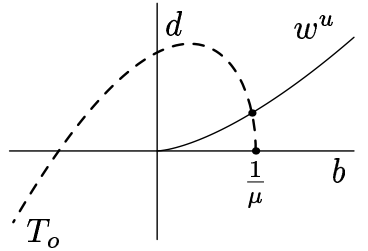

(ii)

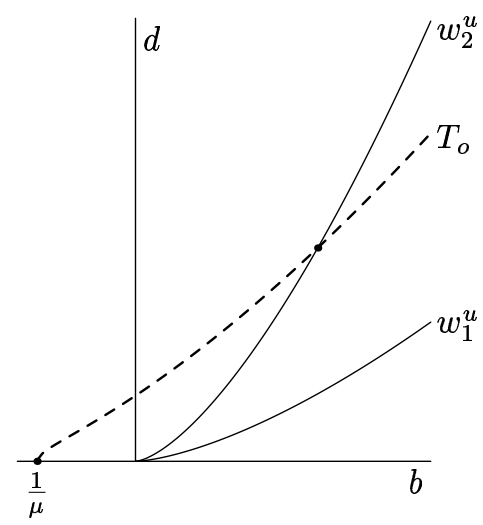

(iv)

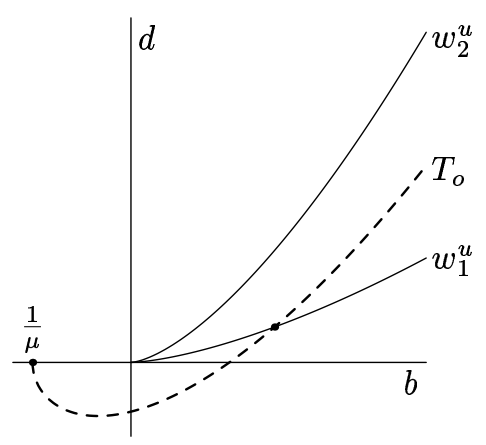

(vi)

Figure 3: The various possible relative positions of $T_{o}$ and $w^{u}$ of Lemma 2.3. (i) For all $\eta>0$ there are no intersections. (ii) For all $\eta>0$ there is one intersection. (iii) By varying $\eta, w^{u}$ moves from $w_{1}^{u}$, for which there are two intersections, to $w_{3}^{u}$ (no intersections). In between, a saddlenode bifurcation occurs at $\eta=\eta_{s n}, T_{o}$ and $w_{2}^{u}$ are tangent. (iv) Depending on $\eta$, there are no intersections $\left(w_{1}^{u}\right)$ or there is one intersection $\left(w_{2}^{u}\right)$. (v) One intersection $\left(w_{1}^{u}\right)$, two intersections $\left(w_{2}^{u}\right)$, or no intersections $\left(w_{3}^{u}\right)$. (vi) One intersection $\left(w_{1}^{u}\right)$ or no intersections $\left(w_{2}^{u}\right)$. 


\subsection{Preliminaries}

We linearize around $\left(A_{h}(x), B_{h}(x)\right)$ and, with a small abuse of notation, re-introduce $a(x)$ and $b(x)$ by

$$
\begin{aligned}
& A(x, t)=A_{h}(x)+a(x) e^{\lambda t}, \\
& B(x, t)=B_{h}(x)+b(x) e^{\lambda t},
\end{aligned}
$$

where $(A(x, t), B(x, t))$ is a solution of $(1.3)$ and $\lambda \in \mathbb{C}$. The linearized stability of the homoclinic pulse solution is thus determined by the following 4-dimensional eigenvalue problem

$$
\left\{\begin{aligned}
-\lambda a & =a-3 A_{h}^{2} a-\mu A_{h} b-\mu B_{h} a-a_{x x} \\
-\varepsilon^{2} \tau \lambda b & =2 \eta \varepsilon^{2} B_{h} b-2 \nu A_{h} a-2 \beta A_{h} B_{h} a-\beta A_{h}^{2} b-\varepsilon^{-2} b_{x x} .
\end{aligned}\right.
$$

This equation can be written as a system of first order ODEs by introducing the vector $\phi(x)=$ $(a(x), v(x), b(x), d(x))^{\top}$, with $a_{x}=v$ and $b_{x}=\varepsilon d$ :

$$
\phi_{x}=\mathcal{A}(x ; \lambda, \varepsilon) \phi
$$

where

$$
\mathcal{A}(x ; \lambda, \varepsilon)=\left(\begin{array}{cccc}
0 & 1 & 0 & 0 \\
\lambda+1+3 A_{h}^{2}(x)+\mu B_{h}(x) & 0 & -\mu A_{h}(x) & 0 \\
0 & 0 & 0 & \varepsilon \\
-\varepsilon\left[2 \nu A_{h}(x)+2 \beta A_{h}(x) B_{h}(x)\right] & 0 & \varepsilon\left[\varepsilon^{2} \tau \lambda+2 \eta \varepsilon^{2} B_{h}(x)-\beta A_{h}^{2}(x)\right] & 0
\end{array}\right) .
$$

Taking the limit $|x| \rightarrow \infty$ in $\mathcal{A}(x ; \lambda, \varepsilon)$ yields the constant coefficient matrix

$$
\mathcal{A}_{\infty}(\lambda, \varepsilon)=\left(\begin{array}{cccc}
0 & 1 & 0 & 0 \\
\lambda+1 & 0 & 0 & 0 \\
0 & 0 & 0 & \varepsilon \\
0 & 0 & \varepsilon^{3} \tau \lambda & 0
\end{array}\right)
$$

The eigenvalues and eigenvectors of $\mathcal{A}_{\infty}(\lambda, \varepsilon)$ are given by

$$
\begin{array}{ll}
\Lambda_{1,4}(\lambda, \varepsilon)= \pm \sqrt{\lambda+1}, & E_{1,4}(\lambda, \varepsilon)=\left(1, \Lambda_{1,4}(\lambda, \varepsilon), 0,0\right)^{\top} \\
\Lambda_{2,3}(\lambda, \varepsilon)= \pm \varepsilon^{2} \sqrt{\tau \lambda}, & E_{2,3}(\lambda, \varepsilon)=\left(0,0,1, \Lambda_{2,3}(\lambda, \varepsilon) / \varepsilon\right)^{\top} .
\end{array}
$$

Although the decay of $\mathcal{A}(x ; \lambda)$ to $\mathcal{A}_{\infty}(\lambda)$ is only algebraic in $x$, the essential spectrum $\sigma_{e}$ of the linear eigenvalue problem (3.2), or (3.3), is still determined by values of $\lambda$ for which either one of the eigenvalues $\Lambda_{j}(\lambda)$ of $\mathcal{A}_{\infty}(\lambda)$ is purely imaginary [12], i.e.,

$$
\sigma_{e}=\{\lambda \in \mathbb{C}: \lambda \in(-\infty, 0]\}
$$

(recall that $\tau>0$ ). Note that although $\lambda=0 \in \sigma_{e}$, it can still be interpreted as an eigenvalue, since it corresponds naturally to the (algebraically decaying) eigenfunction of (3.3) given by the derivative of the wave, i.e., $\left(\frac{d}{d x} A_{h}(x), \frac{d}{d x} B_{h}(x)\right)$. The region $\mathcal{C}_{c}$ is defined as the complement of $\sigma_{e}$

$$
\mathcal{C}_{c}:=\mathbb{C} \backslash \sigma_{e}=\{\lambda \in \mathbb{C}:|\lambda|>0, \arg \lambda \in(-\pi, \pi)\}
$$

For $\lambda \in \mathcal{C}_{c}$, the eigenvalues $\Lambda_{j}(\lambda)$ of $\mathcal{A}(\lambda)$ can be ordered as follows,

$$
\operatorname{Re} \Lambda_{1}(\lambda)>\operatorname{Re} \Lambda_{2}(\lambda)>0>\operatorname{Re} \Lambda_{3}(\lambda)>\operatorname{Re} \Lambda_{4}(\lambda) .
$$

Note that the essential spectrum $\sigma_{e}$ coincides with the absolute spectrum $\sigma_{a}$ of (3.3), or (3.2), since $\operatorname{Re} \Lambda_{2}(\lambda)=\operatorname{Re} \Lambda_{3}(\lambda)$ for $\lambda \in \sigma_{e}$ - see [22] for the definition of $\sigma_{a}$. This is natural, since (3.3) admits the reversibility symmetry $x \rightarrow-x$. However, this also implies, in combination with the fact that $\mathcal{A}(x ; \lambda)$ does not converge exponentially, that the Evans function $\mathcal{D}(\lambda)$ associated 
to (3.3) cannot be expected to be smooth near $\sigma_{e}$ ([23] and section 3.5). Note also that the point $\lambda=0$ is part of the essential/absolute spectrum $\sigma_{e}$, since there is no linear damping in the $B$-equation in (1.3). This property of $\sigma_{e}$ makes it far from trivial to conclude nonlinear stability from linearized stability. We do not consider the issue of the nonlinear stability of the pulse $\left(A_{h}(x), B_{h}(x)\right)$ here. In this paper, we focus completely on the spectral stability of $\left(A_{h}(x), B_{h}(x)\right)$, where we define $\left(A_{h}(x), B_{h}(x)\right)$ to be spectrally stable if the part of the discrete spectrum $\sigma_{d}$ of (3.3) outside $\lambda=0$ is in the stable half plane, i.e., if

$$
\sigma_{d} \backslash\{\lambda=0\} \subset\{\lambda \in \mathbb{C}: \operatorname{Re}(\lambda)<0\} .
$$

\subsection{The matrix $\mathcal{A}_{\text {alg }}(x ; \lambda)$ and its solutions}

In the classical construction of an Evans function $\mathcal{D}(\lambda)$ it is assumed (and used) that the matrix $\mathcal{A}(x ; \lambda)$ of an eigenvalue problem of the type (3.3) converges exponentially to its limit $\mathcal{A}_{\infty}(\lambda)$ as $x \rightarrow \pm \infty$. The 4 fundamental solutions $\phi_{i}(x ; \lambda)$ of $(3.3)$ by which $\mathcal{D}(\lambda)$ is defined are described by their limit behavior (for $x \rightarrow \pm \infty$ ) in terms of the exponential solutions of the constant coefficient problem associated to $\mathcal{A}_{\infty}(\lambda)[1,22]$. Such a construction is not immediately possible for this problem, since $\left\|\mathcal{A}(x ; \lambda)-\mathcal{A}_{\infty}(\lambda)\right\|$ only decays algebraically as $x \rightarrow \pm \infty$.

However, the matrix $\mathcal{A}(x ; \lambda)$ does decay exponentially fast (for $x \rightarrow \pm \infty$ ) to the matrix $\mathcal{A}_{\text {alg }}(x ; \lambda)$, that is defined by

$$
\mathcal{A}_{\mathrm{alg}}(x ; \lambda, \varepsilon):=\left(\begin{array}{cccc}
0 & 1 & 0 & 0 \\
\lambda+1+\mu b_{\mathrm{alg}}(|x|) & 0 & 0 & 0 \\
0 & 0 & 0 & \varepsilon \\
0 & 0 & \varepsilon^{3}\left[\tau \lambda+2 \eta b_{\mathrm{alg}}(|x|)\right] & 0
\end{array}\right),
$$

with $b_{\text {alg }}(x)$ as in (2.9). In fact, it follows from Theorem 2.1 and especially (2.13) that there are positive constants $C$ and $K$ such that

$$
\left\|\mathcal{A}(x ; \lambda)-\mathcal{A}_{\text {alg }}(x ; \lambda)\right\|<C e^{-K|x|}
$$

uniformly in $\lambda \in \mathbb{C}$, i.e., $\mathcal{A}(x ; \lambda)$ decays exponentially fast to $\mathcal{A}_{\text {alg }}(x ; \lambda)$ on the fast spatial scale. The approximation $\mathcal{A}_{\text {alg }}(x ; \lambda)$ of $\mathcal{A}(x ; \lambda)$ is only relevant outside the fast region $I_{f}(2.2)$. We will thus only consider it for $|x| \geq 1 / \sqrt{\varepsilon}$. Note that $\left\|\mathcal{A}(x ; \lambda)-\mathcal{A}_{\text {alg }}(x ; \lambda)\right\|$ is exponentially accurate, in $\varepsilon$ as well as in $x$, outside $I_{f}$.

In the construction of the Evans function associated to (3.3), we now let the matrix $\mathcal{A}_{\text {alg }}(x ; \lambda)$ and its solutions play the role that $\mathcal{A}_{\infty}(\lambda)$ and its exponential solutions play in the standard exponential setting. This choice is both motivated by the exponential convergence of $\mathcal{A}(x ; \lambda)$ to $\mathcal{A}_{\text {alg }}(x ; \lambda)$ and the fact that we can explicitly construct all solutions of

$$
\psi_{x}=\mathcal{A}_{\mathrm{alg}}(x ; \lambda) \psi,
$$

i.e., we can explicitly determine the counterparts of the exponential solutions in the standard exponential case. We can use these functions to prescribe the behavior of the solutions of (3.3) with exponential accuracy (outside the fast region $I_{f}$ ) and define an Evans function based on the limit behavior of the solutions of (3.11).

The matrix $\mathcal{A}_{\text {alg }}(x ; \lambda)$ has a block-diagonal structure, and $(3.11)$ is equivalent to two uncoupled second order equations,

and

$$
a_{x x}=\left[\lambda+1-\mu b_{\mathrm{alg}}(|x|)\right] a,
$$

$$
b_{x x}=\varepsilon^{4}\left[\tau \lambda+2 \eta b_{\mathrm{alg}}(|x|)\right] b .
$$


Note that both equations have inherited the reversibility symmetry of (1.1), so we may restrict our attention to the case $x \geq 1 / \sqrt{\varepsilon}>0$. Since $b_{\text {alg }}(x)$ only varies on a slow spatial scale outside $I_{f}(2.9),(3.12)$ can be solved by a WKBJ approach for $\lambda \in \mathcal{C}_{c}(3.8)$.

Lemma 3.1 Let $\lambda \in \mathcal{C}_{c}$ and define for $x>0$

$$
H(X)=H\left(\varepsilon^{2} x\right)=\sqrt{\lambda+1-\frac{6 \mu}{\eta\left(X^{*}+\varepsilon^{2} x\right)^{2}}},
$$

with $X^{*}$ as in (2.10). The solutions of (3.12) are spanned by $a_{ \pm}(x ; \lambda)$, with

$$
a_{ \pm}(x)=A_{ \pm}\left(\varepsilon^{2} x\right) e^{ \pm \int_{0}^{x} H\left(\varepsilon^{2} s\right) d s} .
$$

Moreover, there is a $C>0$ independent of $\varepsilon$ such that

$$
\left|A_{ \pm}\left(\varepsilon^{2} x\right)-\frac{1}{\sqrt{H\left(\varepsilon^{2} x\right)}}\right|<C \varepsilon
$$

for all $x>0$.

This lemma is proved by direct substitution. Note that there are no turning points, i.e., $H(X) \neq$ 0 , since $\lambda+1-\mu b_{\text {alg }}(x)>\lambda+1-\mu b_{0}>0$ for $\lambda \in \mathbb{R} \backslash \sigma_{e}(2.9),(2.4)$. Note also that the solutions $a_{ \pm}(x)$ decay/grow exponentially on the fast spatial scale, and that

$$
\lim _{x \rightarrow \infty} a_{ \pm}(x ; \lambda) e^{\mp \sqrt{\lambda+1} x}=\frac{1+\mathcal{O}(\varepsilon)}{(1+\lambda)^{1 / 4}} e^{ \pm \frac{C_{\infty}}{\varepsilon^{2}}} \neq 0,
$$

with

$$
C_{\infty}=C_{\infty}(\sqrt{\lambda+1})=\int_{0}^{\infty}\left[\sqrt{\lambda+1-\frac{6 \mu}{\eta\left(X^{*}+X\right)^{2}}}-\sqrt{\lambda+1}\right] d X .
$$

Hence, the decay/growth rates of $a_{ \pm}(x)$ relax algebraically on the slow spatial $X=\varepsilon^{2} x$-scale to the decay/growth rates associated to the $\mathcal{O}(1)$ eigenvalues $\Lambda_{1,4}(\lambda)$ of the matrix $\mathcal{A}_{\infty}(\lambda)(3.6)$.

For $\lambda \neq 0$, equation (3.13) defines a Whittaker equation for $W(z)=b(x)$ where $z=$ $2 \sqrt{\tau \lambda}\left(X^{*}+\varepsilon^{2} x\right)$

$$
W_{z z}+\left\{-\frac{1}{4}+\frac{\kappa}{z}+\frac{\frac{1}{4}-\mu^{2}}{z^{2}}\right\} W=0
$$

with $\kappa=0$ and $\mu=\frac{7}{2}[2,26]$.

Lemma 3.2 Let $\lambda \in \mathcal{C}_{c}$ and define $z=2 \sqrt{\tau \lambda}\left(X^{*}+\varepsilon^{2} x\right)$ for $x>0$. The solutions of (3.13) are spanned by $b_{ \pm}(x ; \lambda)$ that are given by

$$
b_{+}(x ; \lambda)=\left[-\frac{120}{z^{3}}+\frac{60}{z^{2}}-\frac{12}{z}+1\right] e^{\frac{z}{2}}, \quad b_{-}(x ; \lambda)=\left[\frac{120}{z^{3}}+\frac{60}{z^{2}}+\frac{12}{z}+1\right] e^{-\frac{z}{2}} .
$$

As $a_{ \pm}(x)$, the solutions $b_{ \pm}(x)$ decay/grow exponentially as $x \rightarrow \infty$, but now on the slow spatial scale,

$$
\lim _{x \rightarrow \infty} b_{ \pm}(x ; \lambda) e^{\mp \varepsilon^{2} \sqrt{\tau \lambda} x}=1 .
$$

The decay/growth rates of $b_{ \pm}(x)$ correspond to the $\mathcal{O}\left(\varepsilon^{2}\right)$ eigenvalues $\Lambda_{2,3}(\lambda)$ of $\mathcal{A}_{\infty}(\lambda)(3.6)$. 
Proof of Lemma 3.2 Since $\kappa=0,(3.16)$ can be transformed into a Bessel equation by introducing $y(\zeta)=W(2 i \zeta) / \sqrt{\zeta}$,

$$
\frac{d^{2} y}{d \zeta^{2}}+\frac{1}{\zeta} \frac{d y}{d \zeta}+\left(1-\frac{\mu^{2}}{\zeta^{2}}\right) y=0
$$

that has the Bessel functions $J_{ \pm \frac{7}{2}}(\zeta)$,

$$
\begin{aligned}
& J_{+\frac{7}{2}}(\zeta)=\sqrt{\frac{2}{\pi \zeta}}\left[\left(\frac{15}{\zeta^{3}}-\frac{6}{\zeta}\right) \sin \zeta-\left(\frac{15}{\zeta^{2}}-1\right) \cos \zeta\right], \\
& J_{-\frac{7}{2}}(\zeta)=-\sqrt{\frac{2}{\pi \zeta}}\left[\left(\frac{15}{\zeta^{3}}-\frac{6}{\zeta}\right) \cos \zeta+\left(\frac{15}{\zeta^{2}}-1\right) \sin \zeta\right]
\end{aligned}
$$

as solutions $[2,26]$. The Bessel functions $J_{ \pm \frac{7}{2}}(\zeta)$ correspond to two independent solutions $W_{ \pm \frac{7}{2}}(z)$ of (3.16) through

$$
W_{+\frac{7}{2}}(z)=\sqrt{\frac{\pi \zeta}{2}} J_{+\frac{7}{2}}(\zeta), \quad W_{-\frac{7}{2}}(z)=i \sqrt{\frac{\pi \zeta}{2}} J_{-\frac{7}{2}}(\zeta)
$$

with $z=\frac{\zeta}{2 i}$, so that

$$
\begin{aligned}
& W_{+\frac{7}{2}}(z)=-\left(\frac{120}{z^{3}}+\frac{12}{z}\right) \sinh \frac{z}{2}+\left(\frac{60}{z^{2}}+1\right) \cosh \frac{z}{2}, \\
& W_{-\frac{7}{2}}(z)=-\left(\frac{120}{z^{3}}+\frac{12}{z}\right) \cosh \frac{z}{2}+\left(\frac{60}{z^{2}}+1\right) \sinh \frac{z}{2} .
\end{aligned}
$$

Finally, we set $b_{+}(x ; \lambda)=W_{+\frac{7}{2}}(z)+W_{-\frac{7}{2}}(z)$ and $b_{-}(x ; \lambda)=W_{+\frac{7}{2}}(z)-W_{-\frac{7}{2}}(z)$.

Corollary 3.3 Let $\lambda \in \mathcal{C}_{c}$. For $x>0$, the solutions of (3.11) are spanned by the 4 vector-valued functions $\psi_{ \pm, a, b}^{+}(x)$, where

$$
\psi_{ \pm, a}^{+}(x ; \lambda)=\left(a_{ \pm}(x ; \lambda), \frac{d a_{ \pm}}{d x}(x ; \lambda), 0,0\right)^{\top}, \quad \psi_{ \pm, b}^{+}(x ; \lambda)=\left(0,0, b_{ \pm}(x ; \lambda), \frac{1}{\varepsilon} \frac{d b_{ \pm}}{d x}(x ; \lambda)\right)^{\top} .
$$

By the reversibility symmetry, the solutions of (3.11) are for $x<0$ spanned by $\psi_{ \pm, a, b}^{-}(x)$, with

$$
\psi_{ \pm, a}^{-}(x ; \lambda)=\left(a_{\mp}(-x ; \lambda),-\frac{d a_{\mp}}{d x}(-x ; \lambda), 0,0\right)^{\top}, \quad \psi_{ \pm, b}^{-}(x ; \lambda)=\left(0,0, b_{\mp}(-x ; \lambda),-\frac{1}{\varepsilon} \frac{d b_{\mp}}{d x}(-x ; \lambda)\right)^{\top} .
$$

Note that $\psi_{+, a, b}^{-}(x ; \lambda) \rightarrow(0,0,0,0)^{\top}$ as $x \rightarrow-\infty$, i.e., the \pm in the subscript of $\psi_{ \pm, a, b}^{ \pm}(x ; \lambda)$ indicates the sign of the exponent of its exponential component.

\subsection{The Evans function $\mathcal{D}(\lambda)$ and its decomposition}

For $\lambda \in \mathcal{C}_{c},(3.3)$ has a two-dimensional space of solutions that approach $(0,0,0,0)^{\top}$ as $x \rightarrow-\infty$, denoted by $\Phi^{-}(x ; \lambda)$, and a two-dimensional space of solutions that approach $(0,0,0,0)^{\top}$ as $x \rightarrow+\infty, \Phi^{+}(x ; \lambda)$. Since $\mathcal{A}_{\text {alg }}(x ; \lambda)$ is an exponentially accurate approximation of $\mathcal{A}(x ; \lambda)(3.10)$, we may conclude, using standard methods for linear ODEs, that $\Phi^{-}(x ; \lambda)$, respectively $\Phi^{+}(x ; \lambda)$, is approximated by the span of $\psi_{+, a}^{-}(x ; \lambda)$ and $\psi_{+, b}^{-}(x ; \lambda)$, resp. $\psi_{-, a}^{+}(x ; \lambda)$ and $\psi_{-, b}^{+}(x ; \lambda)$.

Lemma 3.4 Let $\lambda \in \mathcal{C}_{c}$ and let $\varepsilon>0$ be small enough. The manifold $\Phi^{-}(x ; \lambda)$, respectively $\Phi^{+}(x ; \lambda)$, is spanned by solutions $\phi_{+, a}^{-}(x ; \lambda)$ and $\tilde{\phi}_{+, b}^{-}(x ; \lambda)$, resp. $\phi_{-, a}^{+}(x ; \lambda)$ and $\tilde{\phi}_{-, b}^{+}(x ; \lambda)$, of (3.3), where $\phi_{\mp, a}^{ \pm}(x ; \lambda)$ and $\tilde{\phi}_{\mp, b}^{ \pm}(x ; \lambda)$ are such that

$$
\left\|\phi_{\mp, a}^{ \pm}(x ; \lambda)-\psi_{\mp, a}^{ \pm}(x ; \lambda)\right\|<C e^{\mp K x},\left\|\tilde{\phi}_{\mp, b}^{ \pm}(x ; \lambda)-\psi_{\mp, b}^{ \pm}(x ; \lambda)\right\|<C e^{\mp K x},
$$


for $\pm x \geq 1 / \sqrt{\varepsilon}$ and some $\mathcal{O}(1)$ constants $C, K>0$. Moreover,

$$
\begin{aligned}
& \lim _{x \rightarrow \pm \infty} \phi_{\mp, a}^{ \pm}(x ; \lambda) e^{ \pm \sqrt{\lambda+1} x}=\frac{1+\mathcal{O}(\varepsilon)}{(1+\lambda)^{1 / 4}} e^{-\frac{C_{\infty}}{\varepsilon^{2}}}(1, \pm \sqrt{1+\lambda}, 0,0)^{\top}, \\
& \lim _{x \rightarrow \pm \infty} \tilde{\phi}_{\mp, b}^{ \pm}(x ; \lambda) e^{ \pm \varepsilon^{2} \sqrt{\tau \lambda} x}=(0,0,1, \pm \varepsilon \sqrt{\tau \lambda})^{\top}
\end{aligned}
$$

with $C_{\infty}$ as in (3.15).

The solution $\phi_{+, a}^{-}(x ; \lambda)$ is determined uniquely, while $\tilde{\phi}_{+, b}^{-}(x ; \lambda)$ is part of a one-parameter family, i.e.,

$$
\tilde{\phi}_{+, b}^{-}(x ; \lambda)=\phi_{+, b}^{-}(x ; \lambda)+s \phi_{+, a}^{-}(x ; \lambda),
$$

for some $s \in \mathbb{R}$ and some 'special' $\phi_{+, b}^{-}(x ; \lambda)$. As in the standard exponential case $[5,7]$, the choice of $\phi_{+, b}^{-}(x ; \lambda)$ will enable us to decompose and explicitly compute the Evans function associated to (3.3). This choice is determined by the behavior of $\phi_{+, b}^{-}(x ; \lambda)$ for $x \geq 1 / \sqrt{\varepsilon}$.

Lemma 3.5 Let $\lambda \in \mathcal{C}_{c}$, let $\varepsilon>0$ be small enough and let $\psi_{+, a}^{+}(x ; \lambda)$ and $\psi_{+, b}^{+}(x ; \lambda)$ as defined in Corollary 3.3. There is a uniquely determined analytic transmission function $t_{a}(\lambda, \varepsilon)$ and $\mathcal{O}(1)$ constants $C, K>0$ such that

$$
\left\|\left(\phi_{+, a}^{-}(x ; \lambda)-t_{a}(\lambda) \psi_{+, a}^{+}(x ; \lambda)\right) e^{-\int_{0}^{x} H\left(\varepsilon^{2} s\right) d s}\right\|<C e^{-K x},
$$

for $x \geq 1 / \sqrt{\varepsilon}$, with $H\left(\varepsilon^{2} x\right)$ as in Lemma 3.1. For $\lambda$ such that $t_{a}(\lambda) \neq 0$, there is a uniquely determined $\phi_{+, b}^{-}(x ; \lambda) \subset \Phi^{-}(x ; \lambda)$ such that

$$
\left\|\phi_{+, b}^{-}(x ; \lambda) e^{-\int_{0}^{x} H\left(\varepsilon^{2} s\right) d s}\right\|<C e^{-K x},
$$

for $x \geq 1 / \sqrt{\varepsilon}$. For this solution $\phi_{+, b}^{-}(x ; \lambda)$, there are two additional meromorphic transmission functions, $t_{b}(\lambda, \varepsilon)$ and $t_{c}(\lambda, \varepsilon)$, such that

$$
\left\|\phi_{+, b}^{-}(x ; \lambda)-t_{b}(\lambda) \psi_{+, b}^{+}(x ; \lambda)-t_{c}(\lambda) \psi_{-, b}^{+}(x ; \lambda)\right\|<C e^{-K x},
$$

for $x \geq 1 / \sqrt{\varepsilon}$.

Note that $\psi_{-, b}^{+}(x ; \lambda)$ decays (slowly) as $x \rightarrow \infty$, so that $t_{b}(\lambda)$ measures the (slow) growth of $\phi_{+, b}^{-}(x ; \lambda)$.

Proof Due to the exponential convergence of $\mathcal{A}_{\text {alg }}(x ; \lambda)$ to $\mathcal{A}(x ; \lambda)$ we can apply the arguments of $[1,5,6]$.

The growth for $x \geq 1 / \sqrt{\varepsilon}$ of the solutions of (3.3) is determined by the fastest growing solution of (3.11), i.e., by $\psi_{+, a}^{+}(x ; \lambda)$ (Corollary 3.3). The transmission function $t_{a}(\lambda)$ as defined in (3.21) measures this growth for $\phi_{+, a}^{-}(x ; \lambda)$; it is by construction uniquely determined and analytic as function of $\lambda$ for $\lambda \in \mathcal{C}_{c}$ [1].

The 3-dimensional subspace $\Phi^{w}(x, \lambda)$ of solutions $\phi(x ; \lambda)$ of $(3.3)$ for which there are $\mathcal{O}(1)$ constants $C, K>0$ such that

$$
\left\|\phi(x ; \lambda) e^{-\int_{0}^{x} H\left(\varepsilon^{2} s\right) d s}\right\|<C e^{-K x},
$$

for $x \geq 1 / \sqrt{\varepsilon}$ consists of all $\phi(x ; \lambda)$ that do not grow as $\psi_{+, a}^{+}(x ; \lambda)$. Since $\Phi^{-}(x, \lambda)$ is twodimensional, the intersection $\Phi^{w}(x, \lambda) \cap \Phi^{-}(x, \lambda)$ must be at least one-dimensional. Clearly, 
$\phi_{+, a}^{-}(x ; \lambda) \subset \Phi^{-}(x, \lambda)$ cannot be an element of this intersection if $t_{a}(\lambda) \neq 0$. Thus, for $\lambda$ such that $t_{a}(\lambda) \neq 0$, there must be a one-dimensional subset of $\Phi^{-}(x, \lambda)$, i.e., a one-parameter family of solutions that decay to $(0,0,0,0)^{\top}$ as $x \rightarrow-\infty$, that is also a subset of $\Phi^{w}(x, \lambda)$. The boundary condition as given in Lemma 3.4 determines the 'special choice' $\phi_{+, b}^{-}(x ; \lambda) \subset \Phi^{w}(x, \lambda) \cap \Phi^{-}(x, \lambda)$ uniquely.

For solutions within $\Phi^{w}(x, \lambda)$, the growth (for $x \geq 1 / \sqrt{\varepsilon}$ ) is determined by the slowly growing solution $\psi_{+, b}^{+}(x ; \lambda)$ of $(3.11)$; apart from $\psi_{+, b}^{+}(x ; \lambda), \Phi^{w}(x, \lambda)$ is furthermore spanned by two solutions that are exponentially close to the (decaying) solutions $\psi_{-, b}^{+}(x ; \lambda)$ and $\psi_{-, a}^{+}(x ; \lambda)$ (this again follows from (3.10)). The function $t_{b}(\lambda)$ measures by definition the (slow) growth of $\phi_{+, b}^{-}(x ; \lambda), t_{c}(\lambda)$ measures the slow component of the decay of $\phi_{+, b}^{-}(x ; \lambda)$. The remaining term $\phi_{+, b}^{-}(x ; \lambda)-t_{b}(\lambda) \psi_{+, b}^{+}(x ; \lambda)-t_{c}(\lambda) \psi_{-, b}^{+}(x ; \lambda)$ is exponentially close to $\psi_{-, a}^{+}(x ; \lambda)$, that also decays exponentially (on an $\mathcal{O}(1)$ spatial scale), so that (3.22) follows.

The transmission functions $t_{b, c}(\lambda)$ are by construction analytic for those $\lambda \in \mathcal{C}_{c}$ for which they are defined. However, they may have poles at the zeroes of $t_{a}(\lambda)[5]$, and they are thus meromorphic as function of $\lambda$.

For $\lambda \in \mathcal{C}_{c}$ and $\varepsilon$ small enough, we can now define the (standard [1, 5]) Evans function $\mathcal{D}(\lambda)$ associated to (3.3) by

$$
\mathcal{D}(\lambda, \varepsilon):=\operatorname{det}\left[\phi_{+, a}^{-}(x ; \lambda), \phi_{+, b}^{-}(x ; \lambda), \tilde{\phi}_{-, b}^{+}(x ; \lambda), \phi_{-, a}^{+}(x ; \lambda)\right],
$$

where $\phi_{-, a}^{+}(x ; \lambda)$ and $\tilde{\phi}_{-, b}^{+}(x ; \lambda)$ are given by the natural counterpart of Lemma $3.4 ; \phi_{-, a}^{+}(x ; \lambda)$ is determined uniquely and $\tilde{\phi}_{-, b}^{+}(x ; \lambda)$ can be chosen from a one-parameter family of solutions such that it depends analytically on $\lambda$ for $\lambda \in \mathcal{C}_{c}$. The trace of $\mathcal{A}(x ; \lambda)$ is $0(3.4)$, thus $\mathcal{D}(\lambda)$ does not depend on $x$. Although $\phi_{+, b}^{-}(x ; \lambda)$ may have a pole (as function of $\lambda$ ) at a zero of $t_{a}(\lambda), \mathcal{D}(\lambda)$ is analytic as function of $\lambda$ for $\lambda \in \mathcal{C}_{c}$. To see this, we can define the alternative Evans function $\tilde{\mathcal{D}}(\lambda)$ by replacing $\phi_{+, b}^{-}(x ; \lambda)$ in $(3.23)$ by a $\tilde{\phi}_{+, b}^{-}(x ; \lambda)$ that depends analytically on $\lambda$; $\tilde{\mathcal{D}}$ is thus analytic in $\lambda$ (for $\lambda \in \mathcal{C}_{c}$ ). Clearly, $\tilde{\phi}_{+, b}^{-}(x ; \lambda)$ and $\phi_{+, b}^{-}(x ; \lambda)$ must be related as in $(3.20)$, where $s=s(\lambda)$ will have poles at the singularities of $t_{b}(\lambda)$ and $\phi_{+, b}^{-}(x ; \lambda)$. It follows from $(3.20)$ that $\mathcal{D}(\lambda)=\tilde{\mathcal{D}}(\lambda)$ for all $\lambda \in \mathcal{C}_{c}$ such that $t_{a}(\lambda) \neq 0$. Thus, $\mathcal{D}(\lambda)$ is analytic in $\mathcal{C}_{c}$, the singularities of $\mathcal{D}(\lambda)$ are removable.

Following [5], we can now consider the limit $x \rightarrow \infty$ in (3.23),

$$
\begin{aligned}
\mathcal{D}(\lambda) & =\lim _{x \rightarrow+\infty} \operatorname{det}\left[\phi_{+, a}^{-}(x ; \lambda), \phi_{+, b}^{-}(x ; \lambda), \tilde{\phi}_{-, b}^{+}(x ; \lambda), \phi_{-, a}^{+}(x ; \lambda)\right] \\
& =\lim _{x \rightarrow+\infty} \operatorname{det}\left[t_{a}(\lambda) \psi_{+, a}^{+} e^{-\sqrt{\lambda+1} x}, t_{b}(\lambda) \psi_{+, b}^{+} e^{-\varepsilon^{2} \sqrt{\tau \lambda} x}, \psi_{-, b}^{+} e^{\varepsilon^{2} \sqrt{\tau \lambda} x}, \psi_{-, a}^{+} e^{\sqrt{\lambda+1} x}\right] \\
& =t_{a}(\lambda) t_{b}(\lambda) \operatorname{det}\left[\frac{1+\mathcal{O}(\varepsilon)}{(1+\lambda)^{1 / 4}} e^{\frac{C_{\infty}}{\varepsilon^{2}}} E_{1}(\lambda), E_{2}(\lambda), E_{3}(\lambda), \frac{1+\mathcal{O}(\varepsilon)}{(1+\lambda)^{1 / 4}} e^{-\frac{C_{\infty}}{\varepsilon^{2}}} E_{4}(\lambda)\right] \\
& =4 \varepsilon \sqrt{\tau \lambda} t_{a}(\lambda, \varepsilon) t_{b}(\lambda, \varepsilon)(1+\mathcal{O}(\varepsilon)),
\end{aligned}
$$

by Lemmas 3.4 and 3.5 (and (3.6),(3.14), (3.18) - note that the two exponential terms cancel). Thus, the zeroes of $\mathcal{D}(\lambda)$ are determined by the zeroes of $t_{a}(\lambda)$ and $t_{b}(\lambda)$. As in the standard, exponential, case $[5,7]$ we will find that $t_{b}(\lambda)$ may have a pole at a zero of $t_{a}(\lambda)$, thus $\mathcal{D}(\lambda)$ can be non-zero if $t_{a}(\lambda)=0$.

The transmission function $t_{a}(\lambda)$ is completely associated to the fast dynamics of (3.3), i.e., the $a$-equation in (3.2). In fact, its zeroes are at leading order given by the eigenvalues of the Sturm-Liouville problem

$$
\left(\mathcal{L}_{f}-\lambda\right) a=a_{\xi \xi}+\left[3 a_{0}^{2}(x)-\left(1-\mu b_{0}+\lambda\right)\right] a=0
$$


associated to the stability of the (unstable) standing pulse solution $a_{0}\left(x ; b_{0}\right)$ of the singular limit (1.4) of (1.3). The pulse solution $a_{0}\left(x ; b_{0}\right)$ of (1.4) coincides with the homoclinic solution of the fast reduced system (2.3) given in (2.5). The eigenvalue problem (3.25) has two eigenvalues, $\lambda_{f}^{0}=3\left(1-\mu b_{0}\right)$ and $\lambda_{f}^{1}=0[7]$.

Lemma 3.6 Let $\varepsilon>0$ be small enough. The transmission function $t_{a}(\lambda)$ has a unique zero $\lambda_{a}(\varepsilon)=\lambda_{f}^{0}+\mathcal{O}(\varepsilon)=3\left(1-\mu b_{0}\right)+\mathcal{O}(\varepsilon)$ in $\mathcal{C}_{c}$. It can be written as $t_{a}(\lambda)=\lambda \tilde{t}_{a}(\lambda)$ with $\tilde{t}_{a}(\lambda)$ such that $\lim _{\lambda \rightarrow 0} \tilde{t}_{a}(\lambda)=\tilde{t}_{a}(0)$ exists.

Proof The relation between the first/fast transmission function, here $t_{a}(\lambda)$, and the stability problem that arises in a (fast) singular limit, and especially the fact that all eigenvalues $\lambda_{f}^{j}$ associated to the fast reduced limit problem persist as zeroes of this transmission function, has been established in a more general setting in [5]. Since $t_{a}(\lambda)$ is a measure for the fast dynamics in $(3.3), \phi_{+, a}^{-}(x ; \lambda)$ and $\psi_{+, a}^{-}(x ; \lambda)$ are both exponentially small for $x \leq-1 / \sqrt{\varepsilon}$, and since the $A$-component of the $\left(A_{h}(x), B_{h}(x)\right)$-pulse decays at an exponential rate, the methods in [5] carry over directly to this setting. Moreover, $t_{a}(\lambda)$ can be defined in a neighborhood of $\lambda=0$, i.e., inside a part of $\sigma_{e}$, since the solutions associated to the $a$-component in (3.3) have $\mathcal{O}(1)$ exponential decay/growth rates for all $\lambda$ such that $\lambda>-\left(1-\mu b_{0}\right)(3.12),(2.4)$. Thus, the eigenvalue $\lambda_{f}^{1}=0$ of the fast reduced limit problem also persists and $t_{a}(\lambda)$ must have a zero near $\lambda=0$. The derivative of the wave $\left(\frac{d}{d x} A_{h}(x), \frac{d}{d x} B_{h}(x)\right)$ is an eigenfunction of (3.3) at $\lambda=0$ of which the $\mathcal{O}(1)$ part decays exponentially with an $\mathcal{O}(1)$ rate. Hence, $\left(\frac{d}{d x} A_{h}(x), \frac{d}{d x} B_{h}(x)\right) \in \operatorname{span}\left\{\phi_{+, a}^{-}(x ; \lambda)\right\}$ which implies that $t_{a}(0)=0$ and thus that $t_{a}(\lambda)=\lambda \tilde{t}_{a}(\lambda)$ with $\tilde{t}_{a}(0)$ well-defined.

In the upcoming section it will be shown that $t_{b}(\lambda)$ must have a pole at $\lambda_{a}(\varepsilon)$, i.e., that $\lambda_{a}(\varepsilon)$ is not a zero of $\mathcal{D}(\lambda)$.

Corollary 3.7 Let $\lambda \in \mathcal{C}_{c}$ and let $\varepsilon>0$ be small enough. The zeroes of the Evans function $\mathcal{D}(\lambda, \varepsilon)$, i.e., the eigenvalues associated to (3.3), are determined by the zeroes of the transmission function $t_{b}(\lambda, \varepsilon)$.

\subsection{The NLEP approach for algebraically decaying pulses}

Although we had to adapt the procedure by which we constructed the Evans functions, i.e., we had to introduce the matrix $\mathcal{A}_{\text {alg }}(x ; \lambda)$, the main outcome of our approach, Corollary 3.7 and especially decomposition (3.24), is completely similar to that of the standard case with exponentially decaying pulses $[5,7]$. In this section, we will see that the NLEP method, by which $t_{b}(\lambda)$ will be computed, can also be extended to the case of algebraically decaying pulses. However, the analysis and the resulting expression for $t_{b}(\lambda)$ are much more involved than in the exponential case (see Remark 3.11). Moreover, we will find that $\mathcal{D}(\lambda)$ has a pole of order 2 at $\lambda=0$, while its counterpart in the exponential case is well-defined at $\lambda=0$. Therefore, the structure of $\mathcal{D}(\lambda)$ near $\lambda=0$, and its relation to saddle-node bifurcations (Lemma 2.4), will be studied in more detail in section 3.5 .

To compute $t_{b}(\lambda)$, we first note by the $b$-equation in $(3.2)$ that the $b$-component $b_{+, b}^{-}(x ; \lambda)$ of $\phi_{+, b}^{-}(x ; \lambda)$ must remain constant (at leading order) over the fast interval $I_{f}$. It thus follows from Lemma 3.4, Corollary 3.3, and Lemma 3.2 that

$$
b_{+, b}^{-}(0)=b_{+, b}^{-}\left(-\frac{1}{\sqrt{\varepsilon}}\right)+\mathcal{O}(\varepsilon)=b_{-}\left(+\frac{1}{\sqrt{\varepsilon}}\right)+\mathcal{O}(\varepsilon)=\frac{\Lambda^{3}+6 \Lambda^{2}+15 \Lambda+15}{\Lambda^{3}} e^{-\Lambda}+\mathcal{O}(\varepsilon),
$$


with

$$
\Lambda=\Lambda(\lambda)=\sqrt{\frac{6 \tau \lambda}{\eta b_{0}}}
$$

$\left((2.10)\right.$ with $b(0)=b_{0}+\mathcal{O}(\varepsilon)$, Theorem 2.1). By Lemma 3.5, it also follows that

$$
b_{+, b}^{-}(0)=\frac{\Lambda^{3}-6 \Lambda^{2}+15 \Lambda-15}{\Lambda^{3}} e^{\Lambda} t_{b}(\lambda)+\frac{\Lambda^{3}+6 \Lambda^{2}+15 \Lambda+15}{\Lambda^{3}} e^{-\Lambda} t_{c}(\lambda)+\mathcal{O}(\varepsilon),
$$

which implies that

$$
t_{c}(\lambda)=1-\left(\frac{\Lambda^{3}-6 \Lambda^{2}+15 \Lambda-15}{\Lambda^{3}+6 \Lambda^{2}+15 \Lambda+15}\right) e^{2 \Lambda} t_{b}(\lambda)+\mathcal{O}(\varepsilon)
$$

Remark 3.8 The polynomial $\Lambda^{3}+6 \Lambda^{2}+15 \Lambda+15$ is zero at $\Lambda=-2.32 \ldots, \Lambda=-1.83 \ldots \pm 1.75 \ldots i$. However $\lambda \in \mathcal{C}_{c}$, so that $\arg \Lambda(\lambda) \in\left(-\frac{\pi}{2}, \frac{\pi}{2}\right)(3.8),(3.27)$. Thus, $\Lambda^{3}+6 \Lambda^{2}+15 \Lambda+15 \neq 0$ and $b_{+, b}^{-}(0) \neq 0$ for $\lambda \in \mathcal{C}_{c}(3.26)$. See also Remark 3.13.

As in the standard, exponential, case [5,7], the expression for $t_{b}(\lambda)$ can now be obtained by determining the total change, $\Delta b_{x}$, in the derivative of $b_{+, b}^{-}(x ; \lambda)$ over $I_{f}$. This can be done in two ways: by using the information on $\phi_{+, b}^{-}(x ; \lambda)$ in the slow field and computing $\Delta_{\text {slow }} b_{x}$, and by integrating $b_{x x}$ over the fast field, which yields $\Delta_{\text {fast }} b_{x}$. On the boundaries of $I_{f}, b_{+, b}^{-}(x ; \lambda)$ is given by Corollary 3.3, and Lemmas 3.4 and 3.5. Thus, $\Delta b_{x}=\Delta_{\text {slow }} b_{x}$ with

$$
\begin{aligned}
\Delta_{\text {slow }} b_{x} & =\left[t_{b}(\lambda) \frac{d}{d x} b_{+}(x)+t_{c}(\lambda) \frac{d}{d x} b_{-}(x)\right]_{x=\frac{1}{\sqrt{\varepsilon}}}-\left[\frac{d}{d x} b_{-}(-x)\right]_{x=-\frac{1}{\sqrt{\varepsilon}}} \\
& =\left[t_{b}(\lambda) \frac{d}{d x} b_{+}(x)+\left(1-\left(\frac{\Lambda^{3}-6 \Lambda^{2}+15 \Lambda-15}{\Lambda^{3}+6 \Lambda^{2}+15 \Lambda+15}\right) e^{2 \Lambda} t_{b}(\lambda)\right) \frac{d}{d x} b_{-}(x)+\frac{d}{d x} b_{-}(x)\right]_{x=\frac{1}{\sqrt{\varepsilon}}} \\
& =\left[t_{b}(\lambda)\left(\frac{d}{d x} b_{+}(x)-\left(\frac{\Lambda^{3}-6 \Lambda^{2}+15 \Lambda-15}{\Lambda^{3}+6 \Lambda^{2}+15 \Lambda+15}\right) e^{2 \Lambda} \frac{d}{d x} b_{-}(x)\right)+2 \frac{d}{d x} b_{-}(x)\right]_{x=\frac{1}{\sqrt{\varepsilon}}} \\
& =2 \varepsilon^{2} \sqrt{\lambda \tau}\left(\frac{\Lambda^{3}}{\Lambda^{3}+6 \Lambda^{2}+15 \Lambda+15} e^{\Lambda} t_{b}(\lambda)-\frac{\Lambda^{4}+6 \Lambda^{3}+21 \Lambda^{2}+45 \Lambda+45}{\Lambda^{4}} e^{-\Lambda}\right)
\end{aligned}
$$

at leading order in $\varepsilon$.

In the fast field $I_{f}, A_{h}(x)$ can be approximated by $a_{0}\left(x ; b_{0}\right), B_{h}(x)$ by $b_{0}$ (Theorem 2.1), and $b(x)$ by $b_{+, b}^{-}(0)(3.26)$. Hence, the $a$-equation in (3.2) is up to terms of $\mathcal{O}(\varepsilon)$ given by the inhomogeneous Sturm-Liouville problem

$$
\left(\mathcal{L}_{f}-\lambda a\right)=a_{x x}+\left[3 a_{0}^{2}(x)-\left(1-\mu b_{0}+\lambda\right)\right] a=-\mu b_{+, b}^{-}(0) a_{0}(x)
$$

(3.25). This is the same inhomogeneous limit problem as was encountered in [7], up to the constant $b_{+, b}^{-}(0)$ in the inhomogeneous part. Note that this is not surprising, since the fast reduced limit problem of the 'system with exponential decay' in [7] is identical to the 'system with algebraic decay' considered here. In [7] the $b$-component of the solution of (3.2) was scaled to 1 in $I_{f}$, here we considered $b=b_{+, b}^{-}(0)$ in $I_{f}$ - see also section 3.5. Equation (3.31) can be solved explicitly [7]. To see this, we introduce

$$
\chi=\sqrt{1-\mu b_{0}} x, \tilde{\lambda}=\frac{\lambda}{1-\mu b_{0}}, w(\chi)=-\frac{\sqrt{1-\mu b_{0}}}{\mu b_{+, b}^{-}(0)} a(x), w_{h}(\chi)=\frac{1}{\sqrt{1-\mu b_{0}}} a_{0}(x)
$$

(recall that $\mu \neq 0$ (section 1$), 1-\mu b_{0} \neq 0(2.4)$, and $b_{+, b}^{-}(0) \neq 0$, Remark 3.8), so that $w_{h}(\chi)$ is the positive homoclinic solution of $W_{\chi \chi}=W-W^{3}$ and (3.31) transforms into

$$
\left(\mathcal{L}_{0}-\tilde{\lambda}\right) w=w_{\chi \chi}+\left[3 w_{h}^{2}(\chi)-(1+\tilde{\lambda})\right] w=w_{h}(\chi) .
$$


For all $\tilde{\lambda} \neq 0,3$, the eigenvalues of $\mathcal{L}_{0}$ ([7], section 3.3), there is a uniquely determined solution $w_{\text {in }}(\chi ; \tilde{\lambda})$ of $(3.33)$ that decays for $\chi \rightarrow \pm \infty$. Since $\left(w_{h}, \frac{d}{d \chi} w_{h}\right)_{L^{2}}=0,(3.33)$ can also be solved at $\tilde{\lambda}=0$ (this (non-unique) solution can be determined explicitly [7]). The operator $\mathcal{L}_{0}-\tilde{\lambda}$ is not invertible at $\tilde{\lambda}=3, w_{\text {in }}(\chi ; \tilde{\lambda})$ has a (simple) pole at $\tilde{\lambda}=3([5,7])$. By $(3.32)$ we conclude that the solution $a_{\text {in }}(x ; \lambda)$ of (3.31) exists for all $\lambda \neq \lambda_{f}^{0}$ (Lemma 3.6) and that $a_{\text {in }}(x ; \lambda)$ has a simple pole at $\lambda_{f}^{0}$.

By the same approximations that yielded (3.31), we now find

$$
b_{x x}=-\varepsilon^{2}\left[2\left(\nu+\beta b_{0}\right) a_{0}(x) a_{\mathrm{in}}(x ; \lambda)+\beta b_{+, b}^{-}(0) a_{0}^{2}(x)\right]
$$

as leading order description of the $b$-equation of (3.2) in $I_{f}$ in which all terms in the right hand side are explicitly known. Thus, the fast field analysis yields a leading order approximation of the accumulated change over $I_{f}$ of the $b$-component of $\phi_{+, b}^{-}(x ; \lambda)$ by integrating $b_{x x}$ as given by (3.34) over $I_{f}$, i.e.,

$$
\Delta_{\text {fast }} b_{x}=-\varepsilon^{2} \int_{-\infty}^{\infty}\left[2\left(\nu+\beta b_{0}\right) a_{0}(x) a_{\mathrm{in}}(x ; \lambda)+\beta b_{+, b}^{-}(0) a_{0}^{2}(x)\right] d x+\mathcal{O}\left(\varepsilon^{2} \sqrt{\varepsilon}\right) .
$$

Note that the computation of $\Delta_{\text {fast }} b_{x}$ has been identical to that in [7]. Before we explicitly determine the leading order expression for $t_{b}(\lambda)$ by setting $\Delta_{\text {slow }} b_{x}=\Delta_{\text {fast }} b_{x}$, we observe that the appearance of $a_{\text {in }}(x ; \lambda)$ in $(3.35)$ implies that $t_{b}(\lambda)$ must have a simple pole near $\lambda_{f}^{0}$. Since $\mathcal{D}(\lambda)$ is analytic near $\lambda_{f}^{0}$, it follows from the decomposition (3.24) that this pole lies exactly at $\lambda_{a}(\varepsilon)$, the zero of $t_{a}(\lambda)$ (Lemma 3.6).

We now define

$$
\mathcal{R}(\tilde{\lambda}):=\int_{-\infty}^{\infty} w_{h}(\chi) w_{\text {in }}(\chi ; \tilde{\lambda}) d \chi
$$

and

$$
\mathcal{J}(\tilde{\lambda}):=2 \beta \sqrt{1-\mu b_{0}}-\frac{\mu\left(\nu+\beta b_{0}\right)}{\sqrt{1-\mu b_{0}}} \mathcal{R}(\tilde{\lambda}),
$$

so that, by (3.32), (2.5), and (3.35),

$$
\Delta_{\text {fast }} b_{x}=-\varepsilon^{2} b_{+, b}^{-}(0) \mathcal{J}(\tilde{\lambda})+\mathcal{O}\left(\varepsilon^{2} \sqrt{\varepsilon}\right)
$$

Moreover, we introduce

$$
\begin{aligned}
& \mathcal{P}(\lambda):=\left(\Lambda^{3}+6 \Lambda^{2}+15 \Lambda+15\right)\left(\Lambda^{4}+6 \Lambda^{3}+21 \Lambda^{2}+45 \Lambda+45\right) \\
& \mathcal{Q}(\lambda):=\frac{\Lambda^{3}+6 \Lambda^{2}+15 \Lambda+15}{\Lambda^{4}+6 \Lambda^{3}+21 \Lambda^{2}+45 \Lambda+45} .
\end{aligned}
$$

It can be checked that the two polynomials in the definitions of $\mathcal{P}(\lambda)$ and $\mathcal{Q}(\lambda)$ both have their zeroes for $\Lambda$ in the negative complex half plane, thus neither $\mathcal{P}(\lambda)$ nor $\mathcal{Q}(\lambda)$ has zeroes or poles for $\lambda \in \mathcal{C}_{c}$ (see Remark 3.8).

Lemma 3.9 Let $\lambda \in \mathcal{C}_{c}$ and let $\varepsilon$ be small enough. The transmission $t_{b}(\lambda)$ as defined in Lemma 3.5 is given by

$$
t_{b}(\lambda, \varepsilon)=\frac{\mathcal{P}(\lambda)}{\Lambda(\lambda)^{7}}\left(1-\frac{\sqrt{6}}{\sqrt{\eta b_{0}}} \mathcal{Q}(\lambda) \mathcal{J}(\tilde{\lambda})\right) e^{-2 \Lambda(\lambda)}+\mathcal{O}(\sqrt{\varepsilon}),
$$

with $\Lambda(\lambda), \mathcal{J}(\tilde{\lambda}), \mathcal{P}(\lambda), \mathcal{Q}(\lambda)$ as in (3.27), (3.37), (3.38), (3.39).

Proof The leading order result of (3.40) follows directly from the above calculations. Uniform estimates on the leading order corrections can be obtained in a straightforward fashion (as in $[5,6,8])$. 
Our stability analysis can now be summarized as follows (see Corollary 3.7).

Corollary 3.10 Let $\lambda \in \mathcal{C}_{c}$ and let $\varepsilon$ be small enough. The zeroes of the Evans function $\mathcal{D}(\lambda)$ are at leading order determined by

$$
\mathcal{T}(\lambda):=1-\frac{\sqrt{6}}{\sqrt{\eta b_{0}}} \mathcal{Q}(\lambda) \mathcal{J}(\tilde{\lambda})=0
$$

Remark 3.11 In the exponential case, i.e., in the study of pulses in a Ginzburg-Landau/diffusion

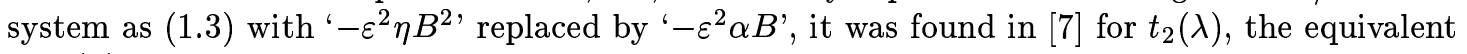
of $t_{b}(\lambda)$, that

$$
t_{2}(\lambda, \varepsilon)=1-\frac{1}{\sqrt{\tau \lambda+\alpha}} \mathcal{J}(\tilde{\lambda})+\mathcal{O}(\sqrt{\varepsilon})
$$

with $\mathcal{J}(\tilde{\lambda})$ as in (3.37). Thus, although the Evans function and the NLEP approach has been successfully extended to the case of pulses with algebraic decay, the endproduct, (3.40), is a more complex expression than its exponential counterpart, with singularity of higher order at $\lambda=0$ - see also Remark 3.13 and Section 3.5.

Note that the only nontrivial component in expression $(3.40)$ for $t_{b}(\lambda)$ is the function $\mathcal{R}(\tilde{\lambda})$. This function can be determined explicitly by using the transformations

$$
P=\sqrt{\tilde{\lambda}+1}, z=\frac{1}{2}\left(1-\frac{\dot{w}_{h}(\chi)}{w_{h}(\chi)}\right), w(\chi)=2^{-\frac{1}{2}(1+3 P)} G(z)\left(w_{h}(\chi)\right)^{P},
$$

to rewrite (3.33) as an inhomogeneous hypergeometric differential equation,

$$
z(1-z) G^{\prime \prime}+(1-2 z)(P+1) G^{\prime}-(P+3)(P-2) G=[z(1-z)]^{-\frac{1}{2}(1+P)},
$$

that can be solved explicitly. Hence, $w_{\text {in }}(\chi)$, the bounded solution of (3.33), can be determined, and $\mathcal{R}(\tilde{\lambda})(3.36)$ can be computed explicitly — see [7] for the details. Thus, the function $\mathcal{R}(\tilde{\lambda})$ can be considered as a known expression.

In the upcoming sections it will be often convenient to consider $\mathcal{R}, t_{b}, \mathcal{T}$, etc. as function of $P$ instead of $\lambda$ or $\tilde{\lambda}$ (with $P$ as in (3.42)). These expressions will be denoted by $\mathcal{R}(P), t_{b}(P)$, $\mathcal{T}(P)$, etc. Moreover, we will use the following properties of $\mathcal{R}(P)$, that have been obtained in [7] (see especially Lemma 4.1 in [7]).

Lemma 3.12 Let $\lambda \in \mathcal{C}_{c}$ and $\mathcal{R}(P)$ as defined in (3.36) with $P=P(\lambda)$ by (3.42), (3.32), then

(i) $\mathcal{R}(P=1)=1, \mathcal{R}^{\prime}(P=1)=R_{1}>0$.

(ii) $\mathcal{R}(P)=R_{2} /(P-2)+\mathcal{O}$ (1) near $P=2, R_{2}<0$.

(iii) $\mathcal{R}(P)=-4 / P^{2}+O\left(1 / P^{4}\right)$ for $P \gg 1$.

(iv) $\mathcal{R}(P)$ has no other poles than $P=2$ for $\lambda \in \mathcal{C}_{c}$.

(v) $\mathcal{R}(P)$ is monotonically increasing for $P>1$ and $P \neq 2$.

(vi) $\mathcal{R}(P)>0$ for $P \in(1,2)$ and $\mathcal{R}(P)<0$ for $P>2$.

Note that $P=1$ corresponds to $\lambda=\tilde{\lambda}=0$, and that $P=2$ to $\lambda=\lambda_{f}^{0}=3\left(1-\mu b_{0}\right)$ (section 3.3), or $\tilde{\lambda}=3$, the critical eigenvalue of $\mathcal{L}_{0}(3.33)$. 
Remark 3.13 By the reversibility symmetry, the eigenfunctions of the eigenvalue problem (3.2) are a priori either even or odd as function of $x$. The zeroes of $\mathcal{D}(\lambda)$ all have $b_{+, b}^{-}(0) \neq 0$ (Remark $3.8)$, thus all eigenfunctions of (3.2) must be even. This can also be seen more explicitly from (3.29), since clearly $t_{c}(\lambda)=1$ (at leading order) if $t_{b}(\lambda)=0$.

This is completely similar to the case of exponential decay, as considered in [7]. Nevertheless, in the algebraic decay case considered here, there can be odd 'resonant eigenfunctions', that are related to the 'resonance poles' of $\mathcal{D}(\lambda)$ [22]. The Evans function $\mathcal{D}(\lambda)$ is a complex valued function that has a two-sheeted Riemann surface as its domain of definition (see also [6, 22, 23]). The essential spectrum $\sigma_{e}$ (3.7) determines a cut in the complex plane, and thus the sheet $\mathcal{C}_{c}=\mathbb{C} \backslash \sigma_{e}(3.8)$, on which $\mathcal{D}(\lambda)$ is uniquely determined. The eigenvalues of (3.2) correspond by construction to the zeroes of $\mathcal{D}(\lambda)$ on this sheet. The second sheet of this Riemann surface corresponds to $\lambda$ such that $\arg \Lambda(\lambda) \in\left(\frac{\pi}{2}, \frac{3 \pi}{2}\right)$ (3.27). Thus, the zeroes of $t_{b}(\lambda)$ coming from $\mathcal{P}(\Lambda)=0$ (3.40), (3.38), i.e., with $\Lambda$ such that $\Lambda^{3}+6 \Lambda^{2}+15 \Lambda+15=0$, define zeroes in $\mathcal{D}(\lambda)$ on the 'wrong', second, sheet (Remark 3.8). Such zeroes are called resonance poles. In principle, a resonance pole may move from the second sheet to $\mathcal{C}_{c}$ as function of a parameter, so that a 'new' eigenvalue appears through $\sigma_{e}$ (this is called an edge bifurcation, see also [5, 22, 8]). This cannot happen to these resonance poles. Note however, that the 'resonant eigenfunctions' associated to the resonant poles are indeed odd, since $b_{+, b}^{-}(0)=0(3.26)$ (this implies by (3.28) that $t_{b}(\lambda)=0$, by (3.31) that $a_{\text {in }}(x) \equiv 0$, and thus by $(3.30)$ and (3.35) that $\left.t_{c}(\lambda)=-1\right)$.

\subsection{The Evans function near $\lambda=0$}

The Evans function $\mathcal{D}(\lambda)$ as defined in Section 3.3 has a pole of order 2 as $\lambda$ approaches 0 .

Corollary 3.14 Let $\lambda \in \mathcal{C}_{c}$ be such that $|\lambda|$ is small, then

$$
\mathcal{D}(\lambda, \varepsilon)=\varepsilon \frac{25 \eta^{3} b_{0}^{3}}{6 \tau^{3}} \tilde{t}_{a}(0)\left[\frac{1}{2} \sqrt{6 \eta b_{0}}-2 \beta \sqrt{1-\mu b_{0}}+\frac{\mu\left(\nu+\beta b_{0}\right)}{\sqrt{1-\mu b_{0}}}\right] \frac{1+\mathcal{O}(\sqrt{\varepsilon}, \sqrt{\lambda})}{\lambda^{2}},
$$

where $\tilde{t}_{a}(0)$ has been defined in Lemma 3.6.

Proof The approximation follows immediately from substitution of the results of Lemmas $3.6,3.9$ and 3.12 into the decomposition (3.24).

The singularity of $\mathcal{D}(\lambda)$ at $\lambda=0$ originates from the scaling of the function $\phi_{+, b}^{-}(x ; \lambda)$ in the definition (3.23) of $\mathcal{D}(\lambda)$. This function is normalized such that its $b$-component approaches 1 as $x \rightarrow-\infty$ (Lemma 3.4, (3.18)). As a consequence, the value of this $b$-component near $x=0$, $b_{+, b}^{-}(0)$, has a singularity near $\lambda=0(3.26)$. This singularity enters into the expression for $\mathcal{D}(\lambda)$ (even twice, since $\tilde{\phi}_{-, b}^{+}(x ; \lambda)$ has been scaled similarly).

Nevertheless, the singularity of $\mathcal{D}(\lambda)$ is essential, in the sense that it represents an intrinsic obstruction in the construction of $\mathcal{D}(\lambda)$ at $\lambda=0$. This is caused by the degenerate behavior of the solutions of the $b$-equation (3.13) at $\lambda=0$. The two independent solutions of this equation,

$$
\tilde{b}_{+}(x ; 0)=\tilde{z}^{4}, \quad \tilde{b}_{-}(x ; 0)=\frac{1}{\tilde{z}^{3}}, \text { with } \tilde{z}=X^{*}+\varepsilon^{2} x,
$$

do not decay exponentially, and, more importantly, cannot be obtained as limits of $b_{ \pm}(x ; \lambda)$ (Lemma $3.2,(3.17))$ for $\lambda=0$. Taking $|\lambda|$ small in $(3.17)$ yields that $b_{ \pm}(x ; \lambda)=\tilde{C}_{ \pm} / \tilde{z}^{3}$, at leading order in $\lambda$ and for $x$ bounded (i.e., $\varepsilon^{2} x=\mathcal{O}(1)$ with respect to $\left.|\lambda| \ll \varepsilon\right)$. Thus, the functions 
$b_{ \pm}(x ; \lambda)$ both approach (a multiple of $) \tilde{b}_{-}(x ; 0)$ in the limit $\lambda \rightarrow 0$, which implies that they do not span the solution space of (3.13) at $\lambda=0$. As a consequence, the construction of the Evans function, and in particular that of the transmission functions $t_{b, c}(\lambda)$, breaks down (Lemmas 3.4 and 3.5). Since the limit behavior of $b_{ \pm}(x ; \lambda)$ and thus that of $\phi_{+, b}^{-}(x ; \lambda)$ is normalized (Lemma 3.4, (3.18)), this degeneration can only appear in $\mathcal{D}(\lambda)$ through $b_{+, b}^{-}(0)$, the value of the $b$-component of $\phi_{+, b}^{-}(x ; \lambda)$ in $I_{f}$ (at leading order).

Note that the functions $\tilde{b}_{ \pm}(x ; 0)(3.44)$ can be obtained as limits of independent solutions of (3.13) with $\lambda \neq 0$ since

$$
W_{+\frac{7}{2}}(z)=\frac{1}{1680} z^{4}+\mathcal{O}\left(z^{6}\right),
$$

(3.19) for $|z| \ll 1$. Thus, $\tilde{b}_{ \pm}(x ; 0)$ appear in the limit $\lambda \rightarrow 0$ from the scaled Bessel functions $W_{ \pm \frac{7}{2}}(z)$. For $\lambda \neq 0$, neither of the functions $W_{ \pm \frac{7}{2}}(z)$ decays to 0 as $z \rightarrow \infty$, therefore they have not been used in the construction of $\mathcal{D}(\lambda)$ for $\lambda \neq 0$ (the decaying function, $b_{-}(x ; \lambda)$, is a linear combination of $\left.W_{ \pm \frac{7}{2}}(z)\right)$.

Conceptually, the construction of an Evans function is based on solutions to the linear eigenvalue system that decay, either for $x \rightarrow-\infty$ or for $x \rightarrow+\infty$. Only for such solutions, the essential property of Evans function - zeroes of $\mathcal{D}(\lambda)$ correspond to eigenvalues - is directly encoded in its definition (see (3.23)). Here, we have found that there cannot be a family of independent solutions to (3.3) that can both be used for the construction of $\mathcal{D}(\lambda)$ and be smoothly extended to $\lambda=0$. Hence, the singular behavior of $\mathcal{D}(\lambda)$ in the limit $\lambda \rightarrow 0$ (Corollary 3.14) is not an artifact of our scalings, it is intrinsic to the stability problem. Note that this is typical behavior for an Evans function associated to the stability problem for a pulse with algebraic decay; $\mathcal{D}(\lambda)$ can even have an essential singularity at $\lambda=0$ [23], however, it is also possible that $\mathcal{D}(\lambda)$ is smooth up to $\lambda=0$ [24].

Finally, we note that the saddle-node bifurcation of homoclinic orbits (Lemma 2.4) also reappears in the Evans function. In the standard exponential case, such a saddle-node bifurcation corresponds to a case in which $\mathcal{D}(\lambda)$ has a double zero at $\lambda=0$ (see for instance [7,8]). Due to the singularity of $\mathcal{D}(\lambda)$, the situation here is less standard. Nevertheless, the saddle-node or tangency condition (2.17) also appears in approximation (3.43) of $\mathcal{D}(\lambda)$ near zero. At the saddle-node bifurcation the order of the singularity of $\mathcal{D}(\lambda)$ at $\lambda=0$ is reduced. Moreover, $\mathcal{T}(\lambda)$ (3.41) is defined at $\lambda=0$, and $\mathcal{T}(0)=0$ (3.41) at a saddle-node bifurcation. By Corollary 3.10, $\lambda=0$ can thus still be interpreted as a double eigenvalue - recall that $\lambda=0$ corresponds as eigenvalue to the $x$-derivative of $\left(A_{h}(x), B_{h}(x)\right)$. In fact, the above developed methods can be used to construct the second independent eigenfunction associated to $\lambda=0$ at the saddle-node bifurcation. Due to the appearance of $\tilde{b}_{-}(x ; 0)(3.44)$ in the $b$-component, this eigenfunction will not decay exponentially, but algebraically (like the other eigenfunction, the derivative of $\left.\left(A_{h}(x), B_{h}(x)\right)\right)$.

Corollary 3.15 A saddle-node bifurcation of homoclinic solutions occurs when the linearized eigenvalue problem (3.2) has a double eigenvalue at $\lambda=0$. Vice versa, a second zero-eigenvalue of (3.2) corresponds to a saddle-node bifurcation.

At one side of the bifurcation point, and $\mathcal{O}(\delta)$ close to it for some $0<\varepsilon \ll \delta \ll 1$, two almost identical homoclinic pulse solutions, $\left(A_{h}^{+}(x ; \delta), B_{h}^{+}(x ; \delta)\right)$ and $\left(A_{h}^{-}(x ; \delta), B_{h}^{-}(x ; \delta)\right)$, exist. The eigenvalues of the stability problem associated to these pulses are also close to each other. In principle, one would expect that both pulses should have an $\mathcal{O}(\sqrt{\delta})$ eigenvalue $\lambda_{s n}^{ \pm}$, that merges with $\lambda=0$ at the saddle-node bifurcation (i.e. as $\delta \rightarrow 0$ ). These eigenvalues can be obtained directly from the equation $t_{b}(\lambda, \varepsilon)=0$ by an expansion in $\delta$, which yields

$$
\lambda_{s n}^{ \pm}= \pm C \sqrt{\delta}+\mathcal{O}(\delta),
$$


with some explicitly computable constant $C \neq 0$ (see section 4.2 for various examples of calculations of this type). Thus, one of the pulses $\left(A_{h}^{ \pm}(x ; \delta), B_{h}^{ \pm}(x ; \delta)\right)$ would have a $\lambda_{s n}^{ \pm}$with $\arg \lambda=\pi$. However, this $\lambda_{s n}^{ \pm}$is inside the essential spectrum $\sigma_{e}$ of the linear stability problem (3.3). Thus, due to the fact that $\sigma_{e}$ reaches up to, and includes $\lambda=0$ (3.7), one of the pulses $\left(A_{h}^{ \pm}(x ; \delta), B_{h}^{ \pm}(x ; \delta)\right)$ does not have an eigenvalue near 0 . As a consequence, one of the $\left(A_{h}^{ \pm}(x ; \delta), B_{h}^{ \pm}(x ; \delta)\right)$ has an even number of eigenvalues, the other odd.

\section{Stability of the homoclinic patterns}

In this section we employ the Evans function machinery developed in section 3 to study the stability of the pulse $\left(A_{h}(x), B_{h}(x)\right)$. We know through Corollaries 3.7 and 3.10 that the eigenvalues of (3.2) correspond to zeroes of $t_{b}\left(P(\lambda)\right.$ ) or even $\mathcal{T}\left(P(\lambda)\right.$ ) (except for the trivial eigenvalue $\lambda_{1} \equiv 0$ that is associated to the derivative of the pulse). As in Theorem 2.1, we distinguish between the cases $\beta=0$ and $\beta \neq 0$ (see also Lemmas 2.2 and 2.3)

\subsection{Stability analysis for $\beta=0$}

We first establish that for $\beta=0$, the eigenvalues cannot enter into $\lambda=0$, and that there is always a unique real unstable eigenvalue for $\mu$ small.

Lemma 4.1 For $\beta=0$, the eigenvalue problem (3.2) has exactly one eigenvalue at $\lambda=0$.

Proof By section 3.5 (especially Corollary 3.15), there can only be a second eigenvalue at $\lambda=0$ if there is a saddle-node bifurcation. This cannot occur for $\beta=0$ (Lemma 2.4).

Lemma 4.2 Let $\beta=0$. There exists a $\mu_{u}>0$ such that the eigenvalue problem (3.2) has exactly one real unstable eigenvalue $\lambda_{u}(\mu, \varepsilon)>0$ for all $\mu$ with $|\mu|<\mu_{u}$. Moreover, $\lim _{\mu \rightarrow 0} \lambda_{u}(\mu, \varepsilon)=3$.

Proof By Corollary 3.10, we only need to consider $\mathcal{T}(P)=0$. For $\beta=0, \mathcal{T}(P)$ can be written as

$$
\mathcal{T}(P)=1+\mu \frac{\sqrt{6} \nu \mathcal{Q}(P)}{\sqrt{\eta b_{0}} \sqrt{1-\mu b_{0}}} \mathcal{R}(P)
$$

Since $\mathcal{R}(P)$ has a singularity at $P=2$ (Lemma 3.12), $\mathcal{T}(P)$ must change sign near $P=2$, i.e., $\tilde{\lambda}=3$ or $\lambda=3\left(1-\mu b_{0}\right)$. Thus, $\mathcal{D}(\lambda)$ must have a zero near $\lambda=3\left(1-\mu b_{0}\right)$ (for $|\mu|$ small). We refer to the proof of Lemma 4.3 in [7] for more details.

The combination of these two results yields that there must always be at least one unstable eigenvalue in the case $\beta=0$. The details of the proof of this statement are identical to those of the proof of Theorem 4.4 in [7]. The main idea behind the proof is that we can use the explicit expression (3.41) and the fact that eigenvalues cannot enter into, or appear from, $\lambda=0$, to show that the number of zeroes of $\mathcal{D}(\lambda)$ in the unstable half plane must always be odd (i.e., eigenvalues cannot appear from/disappear to $\infty$ and can only move into/out of the stable half plane as pairs of complex eigenvalues). Note that it is a priori not excluded that eigenvalues may appear from the essential spectrum $\sigma_{e}$. In fact, we will see in our analysis of cases (v) and (vi) 
of Lemma 2.3 that pairs of complex conjugate eigenvalues may indeed merge into and/or appear from $\sigma_{e}$. Nevertheless, these eigenvalues can only enter the unstable half plane by crossing the imaginary axis away from $\lambda=0$, and thus do not change the parity of the total number of unstable eigenvalues. We again refer to [7] for the details.

Theorem 4.3 Let $\beta=0, \eta>0, \tau>0, \mu \neq 0, \nu>0$ and let $\varepsilon>0$ be sufficiently small. The pulse solution $\left(A_{h}(x), B_{h}(x)\right)$ is spectrally unstable.

\subsection{Stability analysis for $\beta \neq 0$}

For $\beta \neq 0$, most pulse solutions can be regarded as continuations of pulses with $\beta=0$. These pulses will have an unstable eigenvalue related to $\lambda_{0}^{f}$ from the fast reduced limit problem (by varying parameters, as in the proof of Lemma 4.2). However, an additional new real eigenvalue may introduce a mechanism by which all eigenvalues can be brought to the stable half plane (the total number of unstable eigenvalues can become even).

The saddle-node bifurcation (Corollary 3.15) indeed introduces a 'new' zero of $\mathcal{T}(\lambda)$ that a priori may become an extra unstable eigenvalue and thus make the number of unstable eigenvalues even (see section 3.5). Such pulses may be stabilized by a Hopf bifurcation.

Apart from pulse solutions that merge in the limit $\beta \rightarrow 0$ with a pulse solution for $\beta=0$, a pulse $\left(A_{h}(x), B_{h}(x)\right)$ can also diverge as $\beta \downarrow 0$. Such pulse solutions can have an essentially different spectrum. In this scenario it is also possible to have an even number of unstable eigenvalues, and thus that the pulse may be stabilized via a Hopf bifurcation. The analysis below shows that there are indeed pulse solutions which stabilize via this Hopf scenario.

In this section, we study each of the cases distinguished in Lemma 2.3, starting with all situations where $\mu>0$. For $\mu>0$, it can easily be seen that there is at least one real unstable eigenvalue, so that the corresponding pulse solution is spectrally unstable. This occurs in Case (iii) and part of Case (ii) of Lemma 2.3.

Lemma 4.4 Let $\beta \neq 0$. If $\mu>0, t_{b}(P)$ has at least one real zero with $P>2$.

Proof Let $P>2$. Using Lemma 3.12, it can be directly checked that for $P$ close to 2,

$$
t_{b}(P)=\frac{\mathcal{P}(2) \mathcal{Q}(2) e^{-\Lambda(2)}}{\Lambda(2)^{7}} \frac{\sqrt{6} R_{2}\left(\nu+\beta b_{0}\right)}{\sqrt{\eta b_{0}\left(1-\mu b_{0}\right)}} \frac{1}{P-2},
$$

at leading order. It follows that $t_{b}(P)<0$ for $P$ sufficiently close to $2\left(R_{2}<0\right.$, Lemma 3.12). Moreover, a similar analysis yields that $t_{b}(P) \downarrow 0$ as $P \rightarrow \infty(P \in \mathbb{R})$ so that $t_{b}(P)>0$ for $P$ large. Since $t_{b}(P)$ is smooth for $P>2$, it follows that $t_{b}(P)$ has at least one zero for $P>2$.

Theorem 4.5 Let $\beta \neq 0, \mu>0$ and let $\varepsilon>0$ be sufficiently small. The pulse solutions $\left(A_{h}(x), B_{h}(x)\right)$ of (1.3) are spectrally unstable.

Recall that Case (i) of Lemma 2.3 refers to parameter combinations for which no pulse solutions exist. Apart from $\mu>0$ we have to consider pulse solutions of Case (ii) with $\mu<0$. We can determine the eigenvalues of the associated stability problem explicitly in the limit $\eta \downarrow 0$ and in the limit $\eta \gg 0$. 
Lemma 4.6 Let $0<\varepsilon \ll \eta \ll 1$ and $\mu<0, \nu>0, \beta<0$ such that the pulse solution $\left(A_{h}(x), B_{h}(x)\right)$ corresponds to Case (ii) of Lemma 2.3. Apart from the trivial eigenvalue at $\lambda=0$, linearized stability problem (3.3) has a unique eigenvalue $\lambda_{u}(\eta)=3(1+\mu \nu / \beta)+\mathcal{O}(\sqrt{\eta})>0$.

Proof For $0<\eta \ll 1, b_{0}(\eta)$ can be approximated by

$$
b_{0}(\eta)=-\frac{\nu}{\beta}-\frac{\nu^{3 / 2}}{\beta^{2} \sqrt{-6(\beta+\mu \nu)}} \sqrt{\eta}+\mathcal{O}(\eta)
$$

(2.12). Since $\Lambda(P)=\mathcal{O}(1 / \sqrt{\eta}) \gg 1$ (3.27) it follows that $\mathcal{Q}(P)=1 / \Lambda$ at leading order (3.39), so that

$$
\mathcal{T}(P)=1-\frac{2 \beta}{\sqrt{\tau\left(P^{2}-1\right)}}+\mathcal{O}(\sqrt{\eta})
$$

for $P$ not close to 1 or $2(3.41)$. Since $\beta<0, \mathcal{T}(P)$ cannot have a zero for $P$ not close to 1 or 2 . For $P$ close to 2 , we consider the next order approximation of (4.2),

$$
\mathcal{T}(P)=1-\frac{2 \beta}{\sqrt{\tau\left(P^{2}-1\right)}}-\frac{\mu \nu^{3 / 2} \mathcal{R}(P) \sqrt{\eta}}{\sqrt{6 \tau\left(P^{2}-1\right)} \sqrt{-(\beta+\mu \nu)^{3}}} .
$$

This expression can be zero if $\mathcal{R}(P)=\mathcal{O}(1 / \sqrt{\eta})$. By Lemma 3.12 (ii) and (iv) we know that $P=$ $2+p \sqrt{\eta}$ is the only possibility (with $p<0$ if $\mu<0$ ). The result that $\lambda_{u}(\eta)=3(1+\mu \nu / \beta)+\mathcal{O}(\sqrt{\eta})$ follows immediately after substitution.

If $P$ is close to 1 , we introduce $\gamma>0$ by $P^{2}-1=\mathcal{O}\left(\eta^{\gamma}\right)$ so that, by (3.41),

$$
\mathcal{T}(P)=\left\{\begin{array}{lll}
1-\mathcal{O}\left(\eta^{-\frac{\gamma}{2}}\right) \times \mathcal{J}(P) & \text { if } \quad 0<\gamma<1 \\
1-\mathcal{O}\left(\eta^{-\frac{1}{2}}\right) \times \mathcal{J}(P) & \text { if } \quad \gamma \geq 1
\end{array}\right.
$$

However, it can be checked that $\mathcal{J}(P)=\mathcal{O}(1)$ for these values of $\eta$ and $P$, so that we may conclude that the only zero of $\mathcal{T}(P)$ is indeed $\mathcal{O}(\sqrt{\eta})$ close to $P=2$.

Lemma 4.7 Let $\varepsilon>0, \eta \gg 0$ and $\mu<0, \nu>0, \beta<0$ such that $\left(A_{h}(x), B_{h}(x)\right)$ corresponds to Case (ii) of Lemma 2.3. Apart from $\lambda=0$, linearized stability problem (3.3) has a unique real eigenvalue $\lambda_{u}(\eta)=3+\mathcal{O}\left(\eta^{-1 / 3}\right)>0$.

Proof For $\eta \gg 1, b_{0}(\eta)$ is given by

$$
b_{0}(\eta)=\left(\frac{6 \nu}{\eta}\right)^{\frac{1}{3}}+\mathcal{O}\left(\eta^{-\frac{2}{3}}\right)
$$

Substituting this in (3.41), one can do a similar analysis as in the proof of Lemma 4.6 and find that the only possible zero is $P=2+p \eta^{-1 / 3}+$ h.o.t. with $p<0$.

Since there are no saddle-node bifurcations in Case (ii) of Lemma 2.3, i.e., the unstable eigenvalues cannot merge into $\lambda=0$, we conclude that for any $\eta>0$ there must be an odd number of unstable eigenvalues (see [7] and the proof Theorem 4.3). Hence, the pulses associated to Case (ii) of Lemma 2.3 are unstable (Theorem 4.8).

As Theorem 4.5, this result is natural, since these pulses are continuations of pulse solutions with $\beta=0$. In Case (iii) a saddle-node bifurcation occurs. One of the pulse that is created at 
$\eta=\eta_{\mathrm{sn}}$ has an even number of unstable eigenvalues, and cannot be continued to $\beta=0$. Thus, this pulse may in principle be stabilized by the Hopf bifurcation scenario. However, this is not possible since $\mu>0$ in this case (Theorem 4.5).

Pulse solutions that correspond to Case (iv) of Lemma 2.3 can also be regarded as continuations of pulse solutions with $\beta=0$. In this case, one can again prove that there is always an odd number of real unstable eigenvalues.

Theorem 4.8 Let $\varepsilon>0$ be sufficiently small. Every pulse solution $\left(A_{h}(x), B_{h}(x)\right)$ of $(1.3)$ that correspond to one of the cases (i), (ii), (iii) or (iv) of Lemma 2.3 is spectrally unstable.

Neither of the pulse solutions that correspond to Cases (v) or (vi) of Lemma 2.3 can be regarded as continuations of pulse solutions with $\beta=0$. Therefore, their spectrum may differ significantly from those with $\beta=0$. We again consider two limit cases explicitly, $0<\eta \ll 1$ and $\left|\eta-\eta_{\ell}\right| \ll 1$.

Lemma 4.9 Let $0<\varepsilon \ll \eta \ll 1$ and $\mu<0, \nu<0, \beta>0$ such that $\left(A_{h}(x), B_{h}(x)\right)$ corresponds to Cases (v) or (vi) of Lemma 2.3. Apart from $\lambda=0$, (3.3) has two unstable eigenvalues $\lambda_{ \pm}$. Moreover, there are two critical values of $\frac{\tau}{\beta^{2}}$,

$$
\frac{\tau}{\beta^{2}}=S_{ \pm}=\frac{4}{3} \pm \frac{4}{3} \sqrt{6} S_{0} \eta^{\frac{1}{4}}+\mathcal{O}(\sqrt{\eta}), \text { with } S_{0}=\frac{\mu R_{2}}{\sqrt{6}} \sqrt{\left(\frac{-\nu}{\beta+\mu \nu}\right)^{3}}>0
$$

and $R_{2}$ as in Lemma 3.12, such that $\lambda_{ \pm}>\lambda_{f}^{0}=3\left(1-\mu b_{0}\right)>0$ for $0<\frac{\tau}{\beta^{2}}<S_{-},\left|\lambda-\lambda_{f}^{0}\right|=$ $\mathcal{O}\left(\eta^{1 / 4}\right)$ and $\lambda_{ \pm} \in \mathbb{C} \backslash \mathbb{R}$ for $S_{-}<\frac{\tau}{\beta^{2}}<S_{+}$, and $0<\lambda_{ \pm}<\lambda_{f}^{0}$ for $\frac{\tau}{\beta^{2}}>S_{+}$.

Thus, as $\frac{\tau}{\beta^{2}}$ is varied, the eigenvalues $\lambda_{ \pm}$cannot travel through the pole of $t_{b}(\lambda)$ at $\lambda=\lambda_{f}^{0}$, i.e., $P=2$. However, by briefly forming a complex pair, they travel around the singularity.

Proof For $0<\eta \ll 1, b_{0}(\eta)$ is given by

$$
b_{0}(\eta)=-\frac{\nu}{\beta}+\frac{(-\nu)^{3 / 2}}{\beta^{2} \sqrt{6(\beta+\mu \nu)}} \sqrt{\eta}+\mathcal{O}(\eta) .
$$

(compare to (4.1)). For $P$ not close to 1 or $2, \mathcal{T}(P)$ is again given by (4.2), thus, since now $\beta>0$, we find the eigenvalue $\lambda_{+}$associated to

$$
P=P_{+}=\frac{\sqrt{4 \beta^{2}+\tau}}{\sqrt{\tau}} .
$$

Note that this result is not valid if $\left|\tau / \beta^{2}-4 / 3\right| \ll 1$ with respect to $\eta$ since it is assumed in the derivation of $(4.2)$ that $P-2=\mathcal{O}(1)$.

As in the proof of Lemma 4.6, it can be shown that $\mathcal{T}(P)$ cannot have zeroes near $P=1$. To consider the case $|P-2| \ll 1$ with respect to $\eta$, we introduce $p$ and $\gamma>0$ by $P=2+p \eta^{\gamma}$. Moreover, we set $\tau=\frac{4}{3} \beta^{2}\left(1+q \eta^{\theta}\right)$, with $\theta \geq 0$, where $\theta=0$ represents the case $\left|\tau / \beta^{2}-4 / 3\right|=$ $\mathcal{O}(1)$. At leading order, $\mathcal{T}(P)$ is now given by

$$
\mathcal{T}(p, \gamma)=\frac{1}{2} q \eta^{\theta}+\frac{2}{3} p \eta^{\gamma}+\frac{S_{0}}{p} \eta^{\frac{1}{2}-\gamma},
$$


with $S_{0}$ as in (4.3). Thus, for $\theta<\frac{1}{4}$ there are two solutions to $\mathcal{T}(P)=0$,

$$
P_{+}=-\frac{3}{4} q \eta^{\theta}+\text { h.o.t, } P_{-}=-2 \frac{S_{0}}{q} \eta^{\frac{1}{2}-\theta}+\text { h.o.t. }
$$

The solution $P_{+}$merges with (4.4) as $\theta \downarrow 0$. The second zero of $\mathcal{T}(P), P_{-}$, is $\mathcal{O}(\sqrt{\eta})$ close to 2 if $\left|\tau / \beta^{2}-4 / 3\right|=\mathcal{O}(1)$. Note that $P_{ \pm}>2$ for $q<0$, and $P_{ \pm}<2$ for $q>0$, which establishes by (3.42), (3.32) the ordering of $\lambda_{ \pm}$as given in the Lemma.

If $\theta=\frac{1}{4}$, the zeroes of $\mathcal{T}(P)$ are at leading order determined by the quadratic equation

$$
\frac{2}{3} p^{2}+\frac{1}{2} q p+S_{0}=0
$$

This equation determines the transition of $P_{ \pm}$, and equivalently $\lambda_{ \pm}$, from being real-valued to complex-valued and the critical values $S_{ \pm}$as given in (4.3). For $\theta>\frac{1}{4}$, i.e., for $\left|\tau / \beta^{2}-4 / 3\right| \ll$ $\eta^{1 / 4}, P_{ \pm}$are determined by $p^{2}=-\frac{2}{3} S_{0}$ and thus complex-valued.

Lemma 4.10 Let $0<\varepsilon<\left|\eta_{\ell}-\eta\right| \ll 1$ and $\mu<0, \nu<0, \beta>0$ such that $\left(A_{h}(x), B_{h}(x)\right)$ corresponds to Case (v) or (vi) of Lemma 2.3.

(v) $(\beta-2 \mu \nu>0)$ For all $\tau / \beta^{2}$ there exists one unique unstable eigenvalue $\lambda_{\ell} \in\left(0, \lambda_{f}^{0}\right)$.

(vi) $(\beta-2 \mu \nu<0)$ There are two eigenvalues $\lambda_{ \pm}$and there is a critical value of $\tau / \beta^{2}$,

$$
\frac{\tau}{\beta^{2}}=S_{\ell}=\frac{5}{2} R_{1}+\mathcal{O}\left(\sqrt{\left|\eta_{\ell}-\eta\right|}\right)
$$

(Lemma 3.12), such that $\lambda_{ \pm} \in\left(0, \lambda_{f}^{0}\right) \subset \mathbb{R}$ for $\tau / \beta^{2}>S_{\ell}$, and $\lambda_{ \pm} \in \mathbb{C} \backslash \mathbb{R}$ for $\tau / \beta^{2}<S_{\ell}$.

Proof We first consider Case (vi), i.e., $\beta-2 \mu \nu<0$, and introduce $\delta$ by $\eta=\eta_{\ell}(1-\delta)$, with $\eta_{\ell}=-6 \beta^{2} \mu+\mathcal{O}(\varepsilon)$ (Lemma 2.3) and $0<\varepsilon \ll \delta \ll 1$, so that by (2.12),

$$
b_{0}=\frac{1}{\delta}\left[\frac{\beta-2 \mu \nu}{\mu \beta}+\mathcal{O}(\delta)\right] \gg 1 .
$$

The zeroes of $\mathcal{T}(P)$ are determined by

$$
\frac{\sqrt{6}}{\sqrt{\eta b_{0}}} \mathcal{J}(P)=\frac{1}{\mathcal{Q}(P)}
$$

(3.41). At $P=1$ we find, using (4.6),

$$
\frac{\sqrt{6}}{\sqrt{\eta b_{0}}} \mathcal{J}(1)=3+\delta+\mathcal{O}\left(\delta^{2}\right)>\frac{1}{\mathcal{Q}(1)}=3 .
$$

At leading order in $\delta,(4.7)$ is equivalent to

$$
2+\mathcal{R}(P)=\frac{1}{\mathcal{Q}\left(\frac{\sqrt{\tau}}{\beta} \sqrt{P^{2}-1}\right)} .
$$

It can be checked that the right hand side is a monotonically increasing function of $P$, for $P>1$, and that

$$
\frac{1}{\mathcal{Q}\left(\frac{\sqrt{\tau}}{\beta} \sqrt{P^{2}-1}\right)}=3+\frac{2}{5} \frac{\tau}{\beta^{2}}(P-1)+\mathcal{O}\left((P-1)^{\frac{3}{2}}\right)
$$




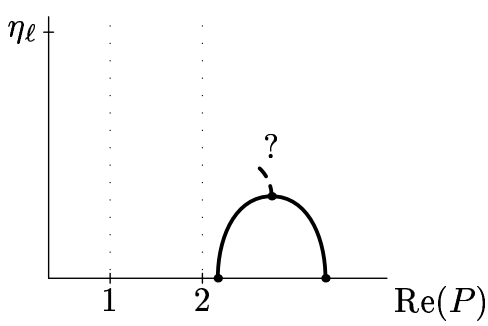

(a)

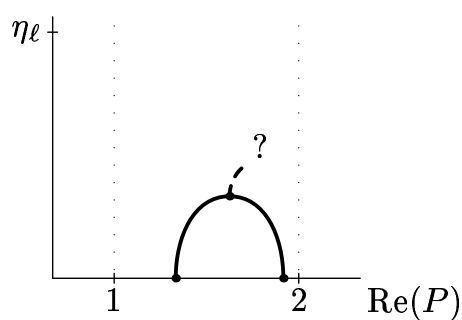

(b)

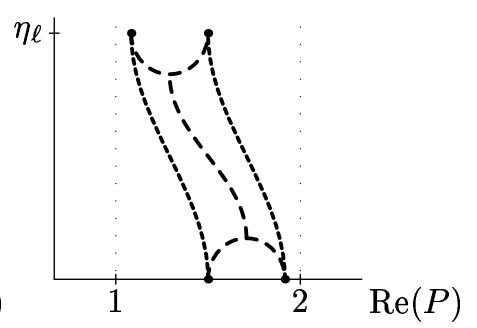

(c)

Figure 4: Possible behavior of the zeroes of $t_{b}(P)$ for a pulse solution of Case (vi) of Lemma 2.3: (a) $0<\tau / \beta^{2}<S_{-}$, the zeroes must merge as function of $\eta$ and form a complex pair; $(b) S_{+}<$ $\tau / \beta^{2}<S_{\ell}$, as in $(a) ;(c) \tau / \beta^{2}>S_{\ell}$, the two real zeroes can either persist or merge temporarily into a complex pair.

for $0<P-1 \ll 1$ (note that $d \mathcal{Q} / d \Lambda(0)=0$ ). Since $\mathcal{R}(P)$ is also monotonically increasing and $\mathcal{R}(P)<0$ for $P>2$ (Lemma 3.12), (4.9) can only have zeroes for $P \in(1,2)$. Moreover,

$$
2+\mathcal{R}(P)=3+R_{1}(P-1)+\mathcal{O}\left((P-1)^{2}\right)
$$

(Lemma 3.12). Thus,

$$
\frac{\sqrt{6}}{\sqrt{\eta b_{0}}} \mathcal{J}(1)>\frac{1}{\mathcal{Q}(1)} \text { and } \lim _{P \uparrow 2} \frac{\sqrt{6}}{\sqrt{\eta b_{0}}} \mathcal{J}(P)>\frac{1}{\mathcal{Q}(2)}
$$

((4.8), Lemma 3.12), and it follows from (4.10) and (4.11) that (4.7) has a degenerate (nontransversal) solution $P_{+}=P_{-} \mathcal{O}(\delta)$ close to $P=1$ for $\tau / \beta^{2}=S_{\ell}$ as in (4.5). Moreover, (4.7) must have two real solutions $P_{ \pm} \in(1,2)$ for $\tau / \beta^{2}>S_{\ell}$ that merge in a pair of complex conjugate solutions when $\tau / \beta^{2}$ decreases through $S_{\ell}$.

The result for Case $(\mathrm{v})(\beta-2 \mu \nu>0)$ runs along the same lines, the main difference is that now

$$
\frac{\sqrt{6}}{\sqrt{\eta b_{0}}} \mathcal{J}(1)=3-\delta+\mathcal{O}\left(\delta^{2}\right)<\frac{1}{\mathcal{Q}(1)}=3,
$$

where $\delta>0$ is now defined by $\eta=\eta_{\ell}(1+\delta)$. As a consequence,

$$
\frac{\sqrt{6}}{\sqrt{\eta b_{0}}} \mathcal{J}(1)<\frac{1}{\mathcal{Q}(1)} \text { and } \lim _{P \uparrow 2} \frac{\sqrt{6}}{\sqrt{\eta b_{0}}} \mathcal{J}(P)>\frac{1}{\mathcal{Q}(2)}
$$

so that (4.7) must have one unique solution $P \in(1,2)$ for all $\tau / \beta^{2}>0$.

Lemmas 4.9 and 4.10 give control over the (unique) Case (vi)-pulse solution of Lemma 2.3 near the boundaries 0 and $\eta_{\ell}$ of its domain of existence. For the Case (v)-pulses, the situation is less simple. Therefore, we first consider the stability of the pulses described by Case (vi).

First, we note that $S_{\ell}>S_{ \pm}(4.3),(4.5)$, which can be checked by direct calculations (or by evaluating $\mathcal{R}(P)[7])$. Thus, we may distinguish a number of different cases, see Figure 4 . In Figure $4(a)$, it is assumed that $0<\tau / \beta^{2}<S_{-}$, and thus there are two real eigenvalues $\lambda_{ \pm}>\lambda_{f}^{0}$, i.e., $P_{ \pm}>2$, for $\eta$ small and no real eigenvalues near $\eta_{\ell}$. We conclude that the eigenvalues $\lambda_{ \pm}$ must merge and form a pair of complex conjugate eigenvalues. For simplicity, we neglect the narrow band $S_{-}<\tau / \beta^{2}<S_{+}$and consider $S_{+}<\tau / \beta^{2}<S_{\ell}$ in Figure $4(b)$. Except for the fact 

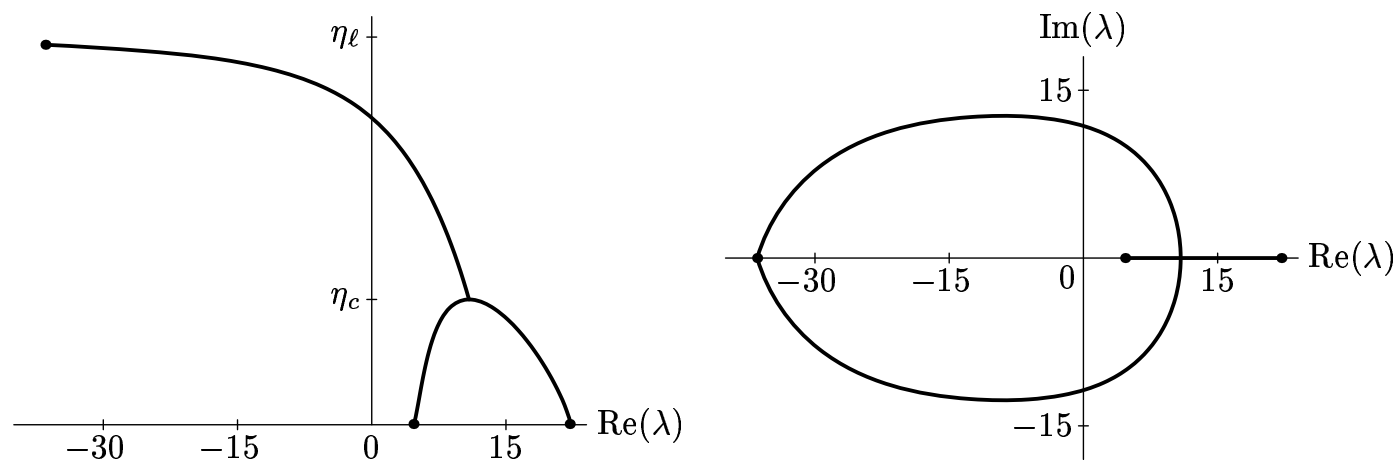

Figure 5: Eigenvalues associated to a pulse $\left(A_{h}(x), B_{h}(x)\right)$ in Case (vi) of Lemma 2.3 as a function of $\eta$ with parameters $\tau=1, \mu=-1 / 4, \nu=-4$ and $\beta=1.9$. There are four critical values of $\eta: \eta_{c}>0$ at which the eigenvalues $\lambda_{ \pm}$merge and form a complex pair, $\eta_{\text {Hopf }}>\eta_{c}$ at which $\lambda_{ \pm}$ cross the imaginary axis so that $\left(A_{h}(x), B_{h}(x)\right)$ is stabilized by a Hopf bifurcation, $\eta_{e}>\eta_{\text {Hopf }}$ at which the eigenvalues disappear into the essential spectrum, and $\eta_{\ell}>\eta_{\text {Hopf }}$ at which the pulse 'disappears to $\infty$.'

that $P_{ \pm} \in(1,2)\left(\lambda_{ \pm} \in\left(0, \lambda_{f}^{0}\right)\right)$ for $\eta$ small the behavior must be similar to that of Figure $4(a)$. In Figure $4(c)$, where $\tau / \beta^{2}>S_{\ell}$, there are two eigenvalues $P_{ \pm} \in(1,2)$, both $\eta$ small and for $\eta$ near $\eta_{\ell}$. Now, as $\eta$ increases, these eigenvalues may either persist, or merge into a complex pair and later merge again to reappear as real zeroes.

Whenever the eigenvalues form a complex pair, the possibility arises that this pair crosses the imaginary axis and thus stabilizes the pulse $\left(A_{h}(x), B_{h}(x)\right)$ via a Hopf bifurcation. Using Mathematica, we can evaluate $\mathcal{R}(P)$, and thus $\mathcal{T}(P)$, explicitly, and can thus determine the eigenvalues of (3.3) for each given set of parameters. By tracing the eigenvalues as function of $\eta$ in the case depicted in Figure 4(c), we observe that the real eigenvalues may persist, and that they may also temporarily form a complex pair, as is sketched in Figure $4(c)$. For parameter combinations as in Figures $4(a)$ and $4(b)$, we indeed observe the formation of a complex pair of eigenvalues. Moreover, we find an open region in $(\tau, \mu, \nu, \beta)$-space in which the complex conjugate pair $\lambda_{ \pm}$crosses the imaginary axis, and the pulse becomes (spectrally) stable.

Theorem 4.11 Let $0<\varepsilon \ll 1$ and $\mu<0, \nu<0, \beta>0, \beta-2 \mu \nu<0$, so that $\left(A_{h}(x), B_{h}(x)\right)$ corresponds to Case (vi) of Lemma 2.3. There is an open region in $(\tau, \mu, \nu, \beta)$-space for which there exists an $\eta_{\mathrm{Hopf}} \in\left(0, \eta_{\ell}\right)$ such that $\left(A_{h}(x), B_{h}(x)\right)$ is spectrally stable for $\eta \in\left(\eta_{\mathrm{Hopf}}, \eta_{\ell}\right)$.

In Figure 5 an explicit example is considered. Note that there is an additional critical value $\eta_{e}$ of $\eta, \eta_{e} \in\left(\eta_{\text {Hopf }}, \eta_{l}\right)$, that is not relevant to the stability of the pulse. At $\eta=\eta_{l}$, the two complex conjugate eigenvalues associated to the stability $\left(A_{h}(x), B_{h}(x)\right)$ merge into the essential spectrum $\sigma_{e}$ - see especially Figure $5(b)$. There are no eigenvalues for $\eta \in\left(\eta_{e}, \eta_{l}\right)$.

Finally, we consider Case (v) of Lemma 2.3, in which there are two pulse solutions, $\left(A_{h}^{ \pm}(x), B_{h}^{ \pm}(x)\right)$, for $\eta \in\left(\eta_{\ell}, \eta_{s n}\right)$, see Figure 3. By definition, $\left(A_{h}^{+}(x), B_{h}^{+}(x)\right)$ exists only for $\eta \in\left(\eta_{\ell}, \eta_{s n}\right)$ and $\left(A_{h}^{-}(x), B_{h}^{-}(x)\right)$ for $\eta \in\left(0, \eta_{s n}\right)$, so that Lemma 4.9 applies to $\left(A_{h}^{-}(x), B_{h}^{-}(x)\right)$ and Lemma 4.10 to $\left(A_{h}^{+}(x), B_{h}^{+}(x)\right)$. Thus, unlike for Case (vi), these lemmas do not give information on the spectrum associated to the pulses at both sides of their domain of existence.

Near $\eta_{s n}$, the number of eigenvalues associated to $\left(A_{h}^{ \pm}(x), B_{h}^{ \pm}(x)\right)$ differs by one (section 3.5). 
Thus, by Lemmas 4.9 and $4.10,\left(A_{h}^{-}(x), B_{h}^{-}(x)\right)$ has an even number of eigenvalues (apart from the trivial eigenvalue at $\lambda=0)$, and $\left(A_{h}^{+}(x), B_{h}^{+}(x)\right)$ an odd number. It immediately follows that $\left(A_{h}^{+}(x), B_{h}^{+}(x)\right)$ cannot be stable (by the arguments that also led to Theorems 4.3 and 4.8).

Either $\left(A_{h}^{+}(x), B_{h}^{+}(x)\right)$ or $\left(A_{h}^{-}(x), B_{h}^{-}(x)\right)$ must have a real eigenvalue that merges with $\lambda=0$ as $\eta \rightarrow \eta_{s n}$ (section 3.5). By evaluating $\mathcal{T}(P)$, we find that there is a 3 -dimensional manifold in the 4-dimensional $(\tau, \mu, \nu, \beta)$ parameter space that distinguishes between cases in which $\left(A_{h}^{+}(x), B_{h}^{+}(x)\right)$ has the real eigenvalue near 0 (see Figure $\left.6(c)\right)$ or $\left(A_{h}^{-}(x), B_{h}^{-}(x)\right)$ does (Figure $6(a, b))$. Note that this manifold can be explicitly determined by a local analysis with $\eta$ near $\eta_{s n}$, as in the proofs of Lemmas 4.9 and 4.10. We refrain from going into the (computationally quite cumbersome) details here.

In the first case, i.e., in which $\left(A_{h}^{-}(x), B_{h}^{-}(x)\right)$ has the eigenvalue near 0 for $\eta$ near $\eta_{s n}$, $\left(A_{h}^{-}(x), B_{h}^{-}(x)\right)$ must also have at least one other real eigenvalue for $\eta$ near $\eta_{s n}$ (since the total number of eigenvalues is even). These two eigenvalues may merge into a complex pair for decreasing $\eta$. However, according to Lemma 4.9 there must also be a pair of unstable real eigenvalues as $\eta$ approaches 0 . Thus, the situation is similar to that sketched in Figure $4(c)$. Nevertheless, it is in principle possible that there is a region $\left(\eta_{\mathrm{Hopf}, 1}, \eta_{\mathrm{Hopf}, 2}\right) \subset\left(0, \eta_{s n}\right)$ in which the imaginary parts of all eigenvalues are negative, i.e., the pulse stabilizes by a Hopf bifurcation, but later destabilizes again by another Hopf bifurcation. We have evaluated $\mathcal{T}(P)$ for many Case (v) parameter combinations, but did not find such a subregion of stability. In Figure $6(c)$ the graphs of the (real values of the) eigenvalues are plotted as function of $\eta$ for a typical situation. Note that the stability problem associated to $\left(A_{h}^{-}(x), B_{h}^{-}(x)\right)$ has one real eigenvalue for all $\eta \in\left(\eta_{l}, \eta_{s n}\right)$.

In the other case, the two real unstable eigenvalues associated to $\left(A_{h}^{-}(x), B_{h}^{-}(x)\right)$ that must exist for $\eta$ near 0 (Lemma 4.9), may also merge into a pair of complex conjugate eigenvalues as $\eta$ increases - see Figure $6(a)$. As in Case (vi), it is found that this pair may move into the stable half plane, i.e. that there is critical value $\eta_{\text {Hopf }}$ of $\eta$ such that $\left(A_{h}^{-}(x), B_{h}^{-}(x)\right)$ is (spectrally) stable for $\eta \in\left(\eta_{\text {Hopf }}, \eta_{s n}\right)$ - see Theorem 4.12. As $\eta \uparrow \eta_{s n}$, the pair of eigenvalues has a welldefined limit $\lambda_{s n}^{ \pm} \subset \mathbb{C} \backslash \mathbb{R}$, see Figure $6(b)$. Thus, the spectrum associated to $\left(A_{h}^{+}(x), B_{h}^{+}(x)\right)$ must have at least 3 eigenvalues for $\eta$ near $\eta_{s n}$, a pair of complex conjugate eigenvalues near $\lambda_{s n}^{ \pm}$, and the eigenvalue near 0 . As $\eta$ decreases, the complex conjugate pair merges into the essential spectrum at a certain critical $\eta_{e} \in\left(\eta_{l}, \eta_{s n}\right)$ - see Figure $6(a, b)$. The other branch persists, so that there is one (real, unstable) eigenvalue for $\eta \in\left(\eta_{l}, \eta_{e}\right)$.

Theorem 4.12 Let $0<\varepsilon \ll 1$ and $\mu<0, \nu<0, \beta>0, \beta-2 \mu \nu>0$, so that there are two pulse solutions $\left(A_{h}^{ \pm}(x), B_{h}^{ \pm}(x)\right)$ (Case (v) of Lemma 2.3); $\left(A_{h}^{+}(x), B_{h}^{+}(x)\right)$ exists for $\eta \in\left(\eta_{\ell}, \eta_{s n}\right)$, $\left(A_{h}^{-}(x), B_{h}^{-}(x)\right)$ for $\eta \in\left(0, \eta_{s n}\right)$. The pulse $\left(A_{h}^{+}(x), B_{h}^{+}(x)\right)$ is always unstable. There $i s$ an open region in $(\tau, \mu, \nu, \beta)$-space for which there exists an $\eta_{\mathrm{Hopf}} \in\left(0, \eta_{s n}\right)$ such that $\left(A_{h}^{-}(x), B_{h}^{-}(x)\right)$ is spectrally stable for $\eta \in\left(\eta_{\mathrm{Hopf}}, \eta_{s n}\right)$.

Acknowledgements The work of GH was supported by NWO grant MEERVOUD 632000.002.

\section{References}

[1] J. Alexander, R. Gardner, and C. Jones. A topological invariant arising in the stability analysis of travelling waves. J. Reine Angew. Math., 410:167-212, 1990.

[2] G. I. Andrews, R. Askey, and R. Roy. Special Functions. Number 71 in Encyclopedia of Mathematics and its Applications. Cambridge University Press, 1999. 


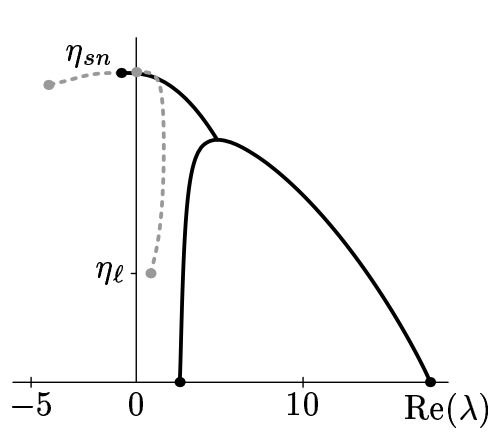

(a)

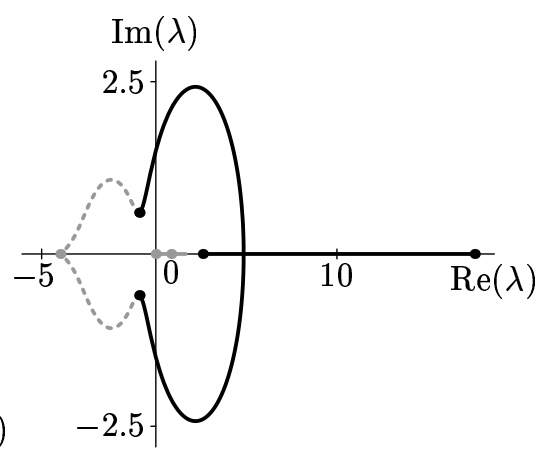

(b)

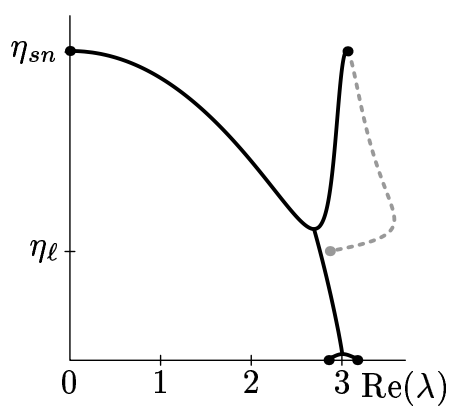

$(c)$

Figure 6: The orbits of the eigenvalues associated to the two pulses $\left(A_{h}^{ \pm}(x), B_{h}^{ \pm}(x)\right)$ in Case (v) of Lemma 2.3 as functions of $\eta$ in two typical situations. The dotted curves denote the eigenvalues associated to $\left(A_{h}^{+}, B_{h}^{+}\right)$, which exist for $\eta \in\left(\eta_{\ell}, \eta_{s n}\right)$, the black curves denote the eigenvalues associated to $\left(A_{h}^{-}, B_{h}^{-}\right)\left(\eta \in\left(0, \eta_{s n}\right)\right)$. Figures $(a)$ and $(b)$ depict the eigenvalues for parameters $\tau=1, \mu=-1 / 4, \nu=-1 / 2, \beta=2$, in which the pulse $\left(A_{h}^{-}, B_{h}^{-}\right)$is stable for $\eta \in\left(\eta_{\text {Hopf }}, \eta_{s n}\right)$. In Figure $(c), \tau=6, \mu=-1 / 4, \nu=-1 / 2, \beta=2$, and both pulses are unstable for all $\eta$.

[3] P. Coullet and S. Fauve. Propagative phase dynamics for systems with Galilean invariance. Phys. Rev. Lett., 55:2857-2859, 1985.

[4] G. Dewel, S. Métens, M. F. Hilali, P. Borckmans, and C. B. Price. Resonant patterns through coupling with a zero mode. Phys. Rev. Lett., 74:4647-4650, 1995.

[5] A. Doelman, R. A. Gardner, and T. J. Kaper. Large stable pulse solutions in reactiondiffusion equations. Indiana Univ. Math. J., 50(1):443-507, 2001.

[6] A. Doelman, R. A. Gardner, and T. J. Kaper. A stability index analysis of 1-D patterns of the Gray-Scott model. Mem. Amer. Math. Soc., 155(737):xii+64, 2002.

[7] A. Doelman, G. Hek, and N. Valkhoff. Stabilization by slow diffusion in a real GinzburgLandau system. J. Nonlinear Sci., 14:237-278, 2004.

[8] A. Doelman, D. Iron, and Y. Nishiura. Destabilization of fronts in a class of bistable systems. SIAM J. Math. Anal., 35(6):1420-1450 (electronic), 2004.

[9] B. Dressel, A. Joets, L. Pastur, W. Pesch, E. Plaut, and R. Ribotta. Competition of periodic and homogeneous modes in extended dynamical systems. Phys. Rev. Lett., 88(2):024503, 2002 .

[10] N. Fenichel. Geometric singular perturbation theory for ordinary differential equations. $J$. Differential Equations, 31(1):53-98, 1979.

[11] G. Hek and N. Valkhoff. Pulses in a complex Ginzburg-Landau system: persistence under coupling with slow diffusion. In preparation, 2006.

[12] D. Henry. Geometric theory of semilinear parabolic equations, volume 840 of Lecture Notes in Mathematics. Springer-Verlag, Berlin, 1981.

[13] C. K. R. T. Jones. Geometric singular perturbation theory. In Dynamical systems (Montecatini Terme, 1994), volume 1609 of Lecture Notes in Math., pages 44-118. Springer, Berlin, 1995. 
[14] N. L. Komarova and A. C. Newell. Nonlinear dynamics of sand banks and sand waves. $J$. Fluid Mech., 415:285-321, 2000.

[15] P. C. Matthews and S. M. Cox. Pattern formation with a conservation law. Nonlinearity, $13(4): 1293-1320,2000$.

[16] A. Mielke. The Ginzburg-Landau equation in its role as a modulation equation. In Handbook of dynamical systems, Vol. 2, pages 759-834. North-Holland, Amsterdam, 2002.

[17] J. Norbury, J. Wei, and M. Winter. Existence and stability of singular patterns in a Ginzburg-Landau equation coupled with a mean field. Nonlinearity, 15(6):2077-2096, 2002.

[18] H. Riecke. Self-trapping of traveling-wave pulses in binary mixture convection. Phys. Rev. Lett., 68:301-304, 1992.

[19] H. Riecke. Solitary waves under the influence of a long-wave mode. Physica D, 92(1-2):69-94, 1996.

[20] H. Riecke. Localized structures in pattern-forming systems. In Pattern formation in continuous and coupled systems (Minneapolis, MN, 1998), volume 115 of IMA Vol. Math. Appl., pages 215-229. Springer, New York, 1999.

[21] C. Robinson. Sustained resonance for a nonlinear system with slowly varying coefficients. SIAM J. Math. Anal., 14(5):847-860, 1983.

[22] B. Sandstede. Stability of travelling waves. In Handbook of dynamical systems, Vol. 2, pages 983-1055, Amsterdam, 2002. North Holland.

[23] B. Sandstede and A. Scheel. Evans function and blow-up methods in critical eigenvalue problems. Discrete Contin. Dyn. Syst., 10(4):941-964, 2004.

[24] B. Sandstede and A. Scheel. Absolute instabilities of standing pulses. Nonlinearity, 18(1):331-378, 2005.

[25] B. Sandstede and A. Scheel. Curvature effects on spiral spectra: Generation of point eigenvalues near branch points. Phys. Rev. E, 73:024503, 2006.

[26] N. Temme. Special functions. An introduction to the classical functions of mathematical physics. John Wiley \& Sons, Inc., New York, 1996.

[27] L. Tsimring and I. Aranson. Localised and cellular patterns in a vibrated granular layer. Phys. Rev. Lett., 79:213-216, 1997.

[28] D. M. Winterbottom, P. C. Matthews, and S. M. Cox. Oscillatory pattern formation with a conserved quantity. Nonlinearity, 18(3):1031-1056, 2005. 